\title{
Low Speed Turbulent Boundary Layer Wind Tunnels
}

\author{
Boldes, U., Colman, J., Marañón Di Leo, J. and Delnero, J.S. \\ Boundary Layer \& Environmental Fluid Dynamics Laboratory \\ Aeronautical Department, Engineering Faculty \\ National University of La Plata, \\ Argentina
}

\section{Introduction}

Turbulence is the last great unsolved problem of classical physics. Or so, it goes for a quote, frequently attributed to one of the great modern physicists Albert Einstein, Richard Feynman, Werner Heisenberg, or Arnold Sommerfeld. A humorous fable, also attributed to several of the great ones, goes as follows - As he lay dying, the modern physicist asked God two questions: Why relativity (or quantum mechanics, depending on who is departing), and why turbulence? "I really think", said the famed physicist, "He may have an answer to the first question."

Due to often unnoticeably perturbations, a particular flow starting from given initial and boundary conditions can often progress reaching quite different flow patterns.

It is a fact that most fluid flows are turbulent, and at the same time fluids occur, and in many cases represent the dominant physics, on all macroscopic scales throughout the known universe, from the interior of biological cells, to circulatory and respiratory systems of living creatures, to countless technological devices (all sizes of planes, wind farms, a wide range of structures, buildings, buildings arrays, etc) and household appliances of modern society, to geophysical and astrophysical phenomena including planetary interiors, oceans and atmospheres. And, despite the widespread occurrence of fluid flow, and the ubiquity of turbulence, the "problem of turbulence" remains to this day a challenge to physicists, engineers and fluid dynamics researchers in general.

No one knows how to obtain stochastic solutions to the well-posed set of partial differential equations that govern turbulent flows. Averaging those non linear equations to obtain statistical quantities always leads to more unknowns than equations, and ad-hoc modeling is then necessary to close the problem. So, except for a rare few limiting cases, first-principle analytical solutions to the turbulence conundrum are not possible.

The problem of turbulence has been studied by many of the greatest physicists and engineers of the 19th, 20th and early 21th Centuries, and yet we do not understand in complete detail how or why turbulence occurs, nor can we predict turbulent behavior with any degree of reliability, even in very simple (from an engineering perspective) flow situations. Thus, the study of turbulence is motivated both by its inherent intellectual challenge and by the practical utility of a thorough understanding of its nature. 
Our particular concern is related with the low atmospheric turbulent boundary layer, that is, the part of the surface layer between ground level and a $400 \mathrm{~m}$ height (this last value depends, more or less, upon the criteria of researchers). Inside this range of height most of human activities are undertaken, specially, those associated with fluid flow over airplanes during takeoff and landings, wind farmers, small and medium size unmanned aerial vehicles, buildings and group of buildings, diverse structures - all immersed in a turbulent boundary layer flow. But, in such "random type flow", we could find turbulence structures, which retain their shape and/or vorticity during a time period, named "coherent structures". These coherent structures are responsible for a great part of the momentum and energy exchanges within the boundary layer. Moreover, many of the problems associated with turbulent low Reynolds number aerodynamics are unsteady.

During the last years, the trend for describing unsteady turbulent flow problems by means of numerical simulation methodologies, based on basic building blocks like elemental eddies and vortices, has increased. The objective is to achieve more realistic representations of key aspects of the dynamic pattern of the oncoming turbulent structures. These computational models are very dependent upon the quality and amount of experimental data obtained in real flow processes or at least in representative wind tunnel experiments. It is known that a direct correlation between the instantaneous aerodynamic behavior of wings and bodies interacting with oncoming particular vortex structures cannot be determined with commonly used statistics methods. Unsteady aerodynamics is a flow-pattern dependent phenomenon. During real flow experiences within a given time record, numerous turbulent structures may go by.

One interesting finding about turbulence was that along with the path to turbulence, very diverse flows run through similar foreseeable phases exhibiting particular predictable pattern characteristics. Turbulent flow patterns often reveal a remarkably self-similar organization. It seems reasonable to hypothesize a correlation between a limited number of particular flow structures and the diffusion transport and mixing behavior of the flow. This picture leads to the known low dimensional approaches. A major issue is how to detect recognize and extract the flow patterns of the turbulent structures governing the flow.

In particular aerodynamic problems, the most representative turbulent structures immersed in the oncoming wind must be previously identified in order to reproduce them in wind tunnel experiments. A main objective in unsteady boundary layer wind tunnel aerodynamics is the realistic reproduction of the dynamic response of a body to oncoming individual turbulent structures immersed in the approaching wind. It is a complex problem, associated with the various space and time scales of the turbulent flow structures. It is known that flying through turbulence changes the aerodynamic forces increasing overall drag and fuel consumption. Nevertheless it is worth to mention that in some cases, a wing submitted to a particular vortex structure embedded in the approaching wind producing intense turbulent velocity fluctuations may only experience an instantaneous Reynolds stresses enhancement without significant changes in the lift forces. The receptivity of twodimensional laminar boundary layers on the curved surface of an airfoil passing through usual atmospheric turbulent free-stream vortices should be considered. It is important to point out that the boundary-layer receptivity to external perturbations characterizes the laminar-turbulent transition problem and therefore the local generation of vortex structures. At first, the dynamic and geometric characteristics of the usually invisible flow pattern of the relevant turbulent structures associated with a particular aerodynamic problem in real flow experiments should be identified. In boundary layer wind tunnel experiments 
adequate inflow turbulence generating mechanisms should be developed in order to obtain an acceptable reproduction. Moreover, despite many years researching turbulent structures, no general detection procedures have been found.

Considering the arguments previously exposed, the study of fluid flows in general and turbulent ones in specific, is necessary to have experimental equipment and computational capability. In the case of turbulent flows and turbulent boundary layer type flows, the necessity of wind tunnels are of upmost importance, together with the possibility to take "in situ" measurements, in order to check the data obtained using the wind tunnel and to also feed the researchers with good "in situ" data in order to reproduce, as closely as possible, the real situation in the wind tunnel. Our concern is on low speed wind tunnels, which are capable to simulating as close as possible, the windy conditions of the lower atmospheric turbulent boundary layer, in particular, coherent structures which are dominant regarding the transport phenomena "modulation", known as boundary layer wind tunnels. It could be of closed circuit or open circuit types.

If we wish to carry out a good job, it will be necessary to perform experiments as close to real conditions as possible (in many cases a lot of experiments), which could be complemented with computational techniques (CFD), but the first ones are almost impossible to avoid. Precisely, CFD is validated with experimental data which could be from wind tunnel experiments reproducing previously known real scenario previously known from "in situ" visualizations and measurements.

In that way, some researchers are interested in the overall flow conditions of wings (and also airfoils) others may focus on small aerial vehicles while others may study the aerodynamics of wing components like flaps, spoilers, etc.

The oncoming turbulent structures immersed in the wind may exhibit very different scales. These scales are usually related to characteristic dimension of the wings and/or airfoils, for example, the chord.

Such turbulent free flow, shape the turbulent boundary layer over the body which researchers wish to manage, with the aim to achieve one (or more) of the following goals: Enhance of the local and/or global lift coefficient, enhance of the maximum lift coefficient, promote or delay the transition, delay the stall, drag reduction or aerodynamic efficiency enhancement. This part of fluid dynamics is known as flow control and is one of the most important branches of current fluid dynamics research in the world.

We could use passive or active devices to attain flow control.

In many cases of interest, for example, wind turbine rotor blades, the Reynolds number based upon the mean free stream velocity and the blade mean chord is of the order or less than $10^{6}$. The aerodynamics for such Reynolds numbers (or lesser) is called low Reynolds number aerodynamics. Following the example cited above, the rotor blade will work under a turbulent free stream flow, at least, on windy days. The "associated" aerodynamic branch is known as low Reynolds number aerodynamics in turbulent flow.

To summarize, the aim and concern of this chapter is to introduce the reader in the fascinating field of the low speed turbulent boundary layer wind tunnels, turbulent boundary layer flows, coherent structures, flow control passive and active devices, action upon airfoils and wings, and wind engineering phenomena in general.

Study of turbulent flows, are of the most importance in several technological applications: aeronautical, naval, mechanical and structural engineering; internal and external flows; transport phenomena; combustion processes; etc. 
The particular characteristics of a turbulent flow structure are directly associated with the aerodynamics forces which promotes upon bodies immersed in the flow, because the flow pattern changes affect lift and drag forces. Typically, lost of momentum due eddies production and viscous dissipation, are usually founded in aeronautical, naval, internal and external flows applications.

If we pretend to improve or optimize an engineering problem which evolves turbulence, it'll necessary to understand and control, at least, the particular group of turbulent structures that govern such phenomena of interest.

Fluid could flow with predictable instantaneous physical magnitudes, as velocities, density, pressure, temperature, etc. If the initial and boundary conditions remain unaltered in time, the non-turbulent flow properties will be associated with those initial and boundary conditions, becoming also time independent or time predictable (periodic oscillations).

In contrast, the instantaneous turbulent velocities will not depend upon initial and boundary conditions. Generally speaking, those velocities are random type. If we perform a lot of experimental velocities measurements, the flow will remain random, that's, the random nature of the instantaneous turbulent flow velocities are independent of how much measurements we could perform. Precisely, random behavior is the main characteristic of turbulent flow.

If we visualize a turbulent flow we'll observe continuous changes in the flow pattern, as a disordered and confused flow. If the turbulent flow will develop without any imposed restrictions, we called it "full developed turbulent flow". What we intend as imposed restrictions? Well, could be gravitational, buoyancy, centrifugal, viscous, electric, magnetic forces, etc.

For example, if we analyze the flow inside a channel, we couldn't considerer developed flow such eddies which scales are similar to the channel scale, because the flow is hard influenced by the forces which govern the flow inside the channel. In other example, eddies of the propeller wake will not be fully developed flow. With these arguments we conclude that almost none turbulent flow could be considered as fully developed, at least, in scales directly associated with high energy.

Small scale low energy turbulent structures could be assumed as fully developed, if viscous forces are of less importance in the flow.

Despite the global random characteristics of turbulent flows, an experimental deep analysis allows us to detect turbulent structures which preserve its form and/or vorticity for a time period. Those structures exhibit an ordered behavior in contrast with the surrounding flow. Those are known as "coherent structures". Moreover, such coherent structures flows immersed in the global random flow. They are responsible and/or play an important contribution to the transport phenomena in the flow.

Researchers, since '60 to the present found that an important part of the turbulent kinetic energy were associated with those coherent structures.

The modern approach to turbulent study is focused on the identification of the various turbulent structures, in particular, those coherent ones.

The understanding of a turbulent flow field implies, by one side, a global analysis and, by other side, an adequate resolution. Global analysis will help us to recognize the large scale structures and, the adequate resolution, the small scale ones. Therefore the need to perform flow experiments, under controlled situations and, also, "in-situ" experiments. The experiments under controlled situations are carried out with proper wind tunnels, named "boundary layer wind tunnels" or "turbulent boundary layer wind tunnels". 
If we are planning to solve turbulent flows by only computational methods, we'll must validate the results with the help of experimental data. For that reason it is essential to build appropriate wind tunnels, together with their associate experimental equipments. For appropriate we mean a wind tunnel capable to "reproduce" as close as possible, the wind characteristics of the low atmospheric boundary layer and/or any other turbulent type boundary layer.

Different methodologies are employed to process the huge acquired data in order to extract flow structures from measured time series, the classical statistics, quadrant analysis, Wavelets transforms, Proper Orthogonal Decomposition (POD), etc. Also there are various visualization flow techniques, because it's of most importance to "see" how the flow is, that's, the global flow pattern, with the aim to try to identify eddies, its spatial distribution and orientation its time dependent geometry its scales. The usual initial approach is to find position and track the larger vortices.

The resulting data will be very useful and necessary to characterize the turbulent flow pattern and, also, try to identify distinct geometric and dynamic features of the main coherent structures in the flow.

\section{Coherent structures}

Almost all the fluid dynamics researchers are coincident in their opinion about that coherent structures are responsible of the fluid behavior prediction failures employing classical turbulence theories. One of those examples is the use of only mean velocities gradients to describe the turbulent wake of porous bodies, no predicting the secondary maximum.

With the aim of detecting, identifying and examining coherent flow structures, a variety of detection techniques are commonly used in diverse flows (e.g. Bonnet et al., 1998).

On the other hand despite decades of investigation on coherent structures and their characterization no general detection methodology has been established.

Turbulent organized structures have decisive influence upon transport phenomena due their capacity to establish the way to be follow by important fluid mass volumes (See, for example, McWilliams \& Weiss (1994) and Babiano et al (1994)).

A coherent structure could be imagine as a random space region which, for certain amount of time, exhibit some organization degree in, at least, one of their flow properties, that's velocity, vorticity, pressure, density, temperature, etc.

On speaking about "organization" we mean that what happens in one instant in one space point is connected with the behavior of the flow in other time interval and/or another space points. So, a coherent structure moves exhibiting some organization degree. We could imagine the situation like a part of the fluid with random behavior, is transported by the flow, preserving its cohesion. This part of the flow could rotate (which imply a vortical coherent structure) and also could deform, stretch, longing, heating or cooling.

Vortical structures, like eddies, are usually founded in many fluid flows. Sometimes are easily visible, great and well defined scale; sometimes are hard to identify due their small scale and/or their unclear boundaries and, in some occasions, we are unable to distinguish they at a plain sight. Moreover, at the present there wasn't, in the fluid dynamics researchers world, an unified, clear and complete definition of what's a vortex, where it begins and ends.

For example, definitions based on such flow zones where there are vorticity is not precise, because they are unable to distinguish between a zone with non-rotating flow but with high 
shear with those zone where fluid rotates. Also, until the present, researchers had not found a clear boundary between vortex structures and the surrounding turbulent flow.

Conceptually, we could say that coherent structures are:

a. A space zone where vorticity is concentrated as a way that promotes the fluid to follow trajectories which rolls around it.

b. Following the structure movement, it could change its shape (for example, from cylindrical to elliptical), splitting in small structures or merging with neighbor structures becoming bigger vortices or disintegrating.

c. This coherent structures, appears in the flow in an unpredictable way.

Robinson (1991), for example, made the following definition of a coherent structure: "a coherent movement is defined as a tridimensional flow region, upon which at least one fundamental flow magnitude (velocity component; density; temperature; etc) exhibits a significant correlation between itself and/or with other magnitude in a spatial/temporal range bigger than the flow micro-scales".

Hussain (1986), by other side, provides a more restrictive definition: "Coherent structure is a connected mass flow, in turbulent flow, which vorticity is instantaneously correlated in all mass flow spatial extension".

The apparently flow random behavior is due, mainly, to the random size and intensities of the different organized structures which belong to the fluid flow. Coherent structures are, in general, easier to detect in free flows than wall type flows.

Researchers challenge is, precisely, the identification of such coherent structures present in a whole random flow, when such structure belong to a complex velocity, temperature or pressure signal.

\section{Low atmospheric turbulent boundary layer (general remarks)}

In windy conditions, shear stresses are very important from the surface terrain to $300 \mathrm{~m}$ to $400 \mathrm{~m}$ height, becoming the typical boundary layer flow, mainly turbulent. The part of the layer, in direct contact with the surface, is called the viscous sublayer. This layer is characterized by very strong vertical wind shear (change of direction with height). The depth of the viscous sublayer is a few millimeters.

Close to the ground lies a region in which the friction velocity is essentially constant and equal to the value at the surface. This region is known as the surface layer or constant-stress layer. It is above the viscous sublayer and has a typical depth of $20-300 \mathrm{~m} / 400 \mathrm{~m}$. In fact, the viscous sublayer is part of the surface layer and some researchers don't distinguish between them, calling both with the general specification of surface layer.

Very close to the earth's surface the wind velocity is reduced to zero by the drag of surface elements. This takes place in the roughness layer, the depth of which is comparable to the size of the surface roughness elements (grass, houses, group of houses, buildings, woods, etc). The flow above the roughness layer contains small-scale, time-dependent motions, or eddies. Velocities, temperatures, and other state variables may be expressed formally as the sum of the mean variables and eddy variables (velocities, momentum, entropy, etc). That's the classical approach to turbulence study, mentioned above by us.

If we take account that many of the human activities take place inside such layer, it's natural to understand why fluid dynamics researchers try to understand and carefully study the flow characteristics of such region. Woods's induced turbulence; suburban areas; cities; etc, are immersed in such boundary layer turbulent flow. Theoretical and/or computational 
study, only, will not drive them self to obtain good explanation of how the flow is, how are coherent structures in such layer and, subsequently, which are the associated aerodynamic forces.

For that reason we need to perform experiments, which will be "in situ" and wind tunnel ones. Moreover, such wind tunnels must be capable to reproduce, as close as possible, the flow conditions in the surface layer. Such wind tunnels type, are known as turbulent boundary layer wind tunnels.

Due the complexity of the flow in the surface layer, early researchers like Monin and Obukhov (1954), developed a similarity theory with the objective to organize and group the acquired experimental data. The theory aim was the identification of the most important physical parameters and, then, to define dimensionless groups with it. After that, experimental data were used to find functional relations between such dimensionless groups. Once he functional relations are known, they were used as part of a parameterization scheme.

Under this context, the relevant parameters for the surface layer were: momentum flux, buoyancy flux and the dimensionless height above the earth surface. Precisely, this last parameter is the turbulent length scale; due that eddies scales are determined by their distance from the earth surface.

One of the parameters is the Monin-Obukhov length L (see Monin et al, 1954) and, together with the friction velocity $\mathrm{u}^{*}=\left(\tau_{\mathrm{w}} / \rho\right)^{1 / 2}$, was possible to establish a simple relation between mean time turbulent velocities and the dimensionless height $\mathrm{z} / \mathrm{L}$, being for example, one of them:

$$
\left[k z u * \sqrt{\left(-u^{\prime} w^{\prime}\right)}\right](\partial V / \partial z)=\Phi_{M}(z / L)
$$

This function $\Phi_{M}(z / L)$ relates the friction velocity $u^{*}$, the vertical gradient $\partial V / \partial z$ and the shear as a function of $z / L$. Note: $\tau_{w}$ and $\rho$ are the shear over the terrain and air density, respectively.

Also, it's possible to relate the vertical mean velocities profile with the dimensionless $z / z_{0}$, being $z$ the height and $z_{0}$ the "roughness medium height" which will be different if we are dealing with a plane grass field, the sea and/or ocean, suburban areas and urban ones. Such relations are known as logarithmic mean velocities profile and mean velocities power law:

$\mathrm{u}(\mathrm{z})=\left(\mathrm{u}^{*} / \mathrm{k}\right) \ln \left(\mathrm{z} / \mathrm{z}_{0}\right)$ (logarithmic, valid for very short vegetation and neutral atmosphere)

$\mathrm{u}(\mathrm{z}) / \mathrm{U}_{\mathrm{m}}=\left(\mathrm{z} / \mathrm{z}_{0}\right)^{\alpha}$ (potential law, useful for roughness terrain and small roughness terrain and sea)

In both equations $u$ is mean velocity along $\mathrm{x}$-axis (parallel to the floor). In the last equation, the exponent a will vary according the terrain roughness, decreasing proportionally to roughness values.

Monin-Obukhov length, L, is a stability parameter which serve as "indication parameter", that's for example when $z / L<<1$ is valid the logarithmic law (mentioned above).

At this stage the authors doesn't wish to show and/or develop the whole surface layer theory and the similarity one, just only to point out the essential concepts of them, with the purpose to show up the spirit of the design, building and operation of turbulent boundary layer wind tunnels and, subsequently, to display some of the fluid dynamic experiments carried out with their help. 


\section{Turbulent boundary layer wind tunnels at the boundary layer \& environmental fluid dynamics laboratory}

\subsection{Closed circuit wind tunnel}

Since 1984 is operating, at the Aeronautical Department, Engineering Faculty, National University of La Plata, Argentina, the first turbulent boundary layer wind tunnel, closed circuit one. The test section dimensions are $7.5 \mathrm{~m}$ length and $1.4 \times 1 \mathrm{~m}^{2}$ traverse section. The tunnel has a direct current $50 \mathrm{HP}$ motor with their corresponding electronic speed control and 6 blades. The maximum velocity, at the test section, is $20 \mathrm{~m} / \mathrm{s}$. The wind tunnel is equipped, at the begin of the test section, with a honeycomb in order to achieve a flow with directional preference along $\mathrm{x}$-axis (test section) and, after that, a vertical array of aluminum profiles, parallel to the tunnel floor, distributed with a given variable vertical distance between them. Each profile is capable to manually rotate along its longitudinal axis. These arrays serves as turbulence generators which allow to obtain different power law exponents and also the logarithmic law and, also, different turbulence intensities with their corresponding vertical evolution. Roughness elements (parallelepipeds) are distributed over the tunnel floor to achieve the roughness turbulence for different conditions according the real ones in urban, suburban and field scenarios.

Figures 1 and 2 shows the turbulent generators profiles, in vertical array after honeycomb, together with the turbulence generators triangles (after profiles), and details of the test section, included the roughness elements. At the test section we could see the portable Dantec Flowmaster anemometer arm. We use such anemometer to continuously verify the mean velocity stream at the test section. The instantaneous velocities measurements are made with the Dantec Streamline 6 channels hot-wire constant temperature anemometer.

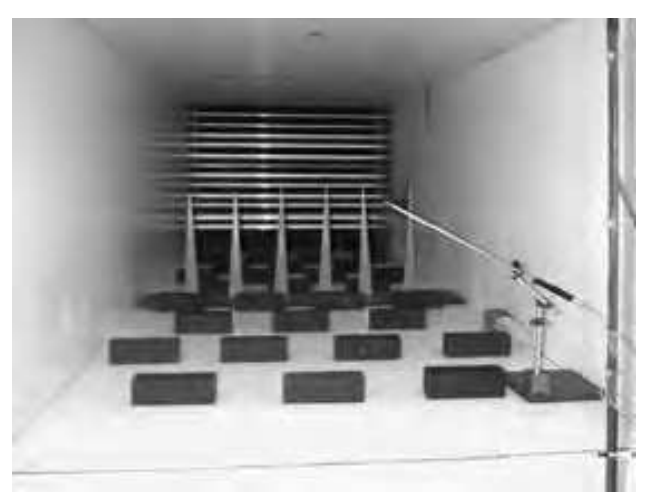

Fig. 1. Triangular mixing spikes 


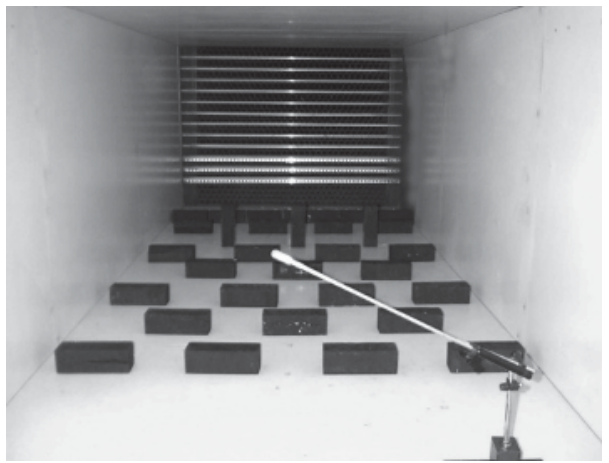

Fig. 2. Roughness elements

Figures 3 and 4 show us, respectively, the external view of the test section, with the 6 channels anemometer and data acquisition PC and a wing model between two double panels, inside the test section.

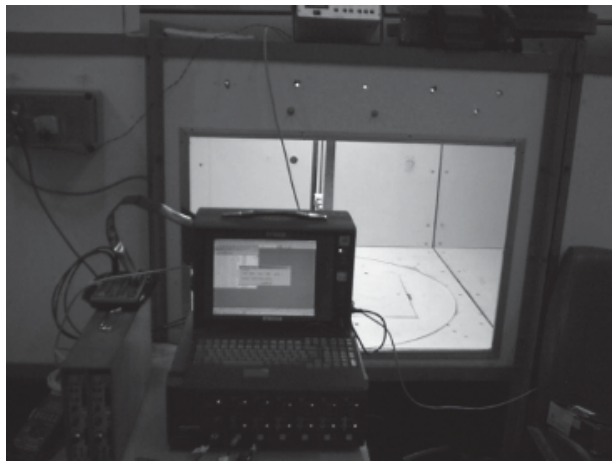

Fig. 3. Test section and Measuring equipment

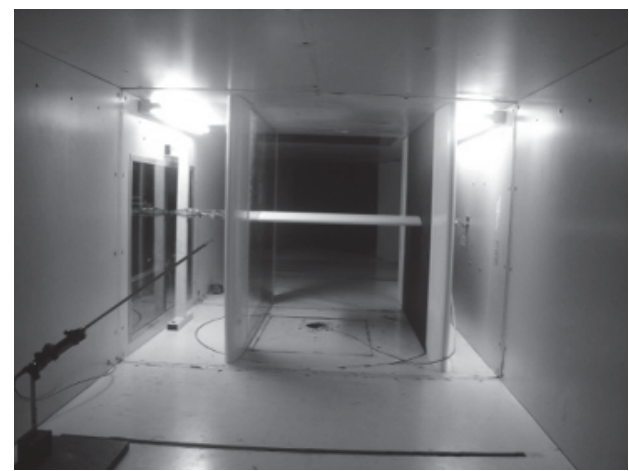

Fig. 4. Test section

Figures 5 and 6 corresponding to typical power law mean velocities distribution vs. height and autocorrelation, respectively. 


\section{Mean velocity profile}

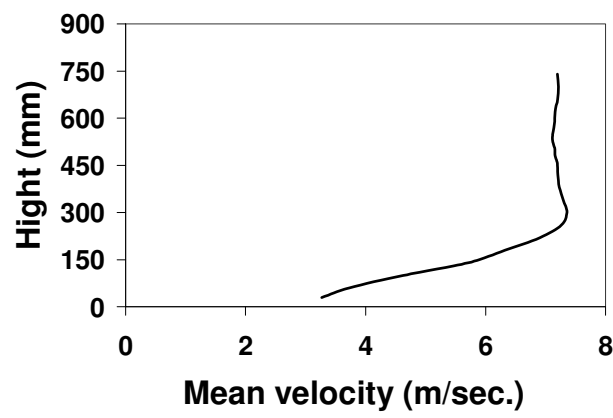

Fig. 5. Mean velocity profile

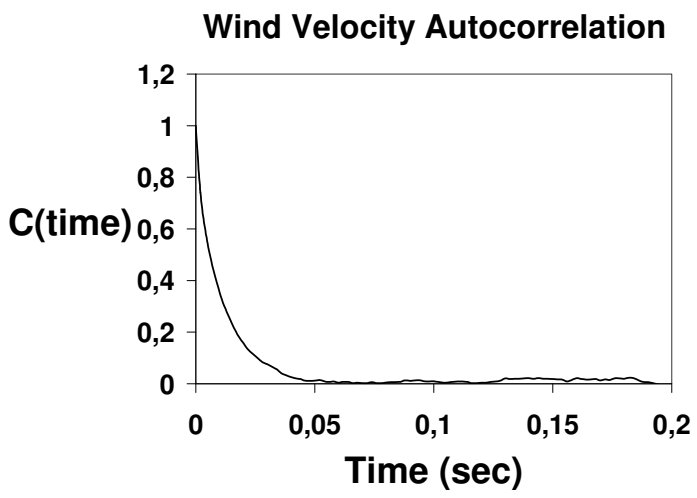

Fig. 6. Wind velocity autocorrelation

Figures 7 and 8 corresponds to typical turbulence intensity distribution vs. height and shear stress distribution vs. height

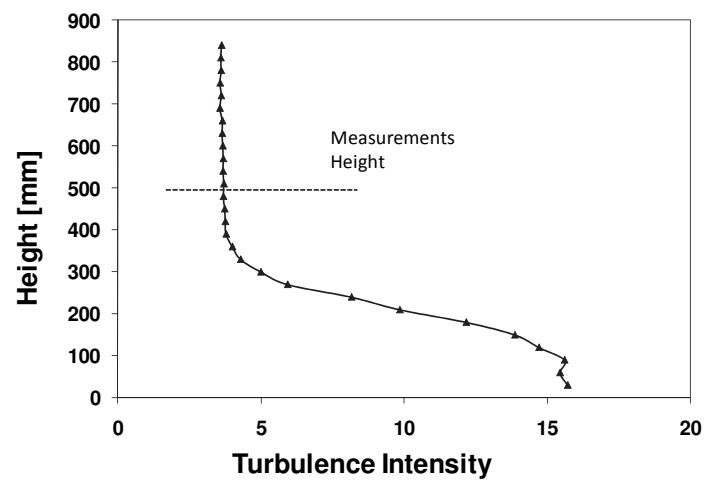

Fig. 7. Turbulence Intensity distribution 


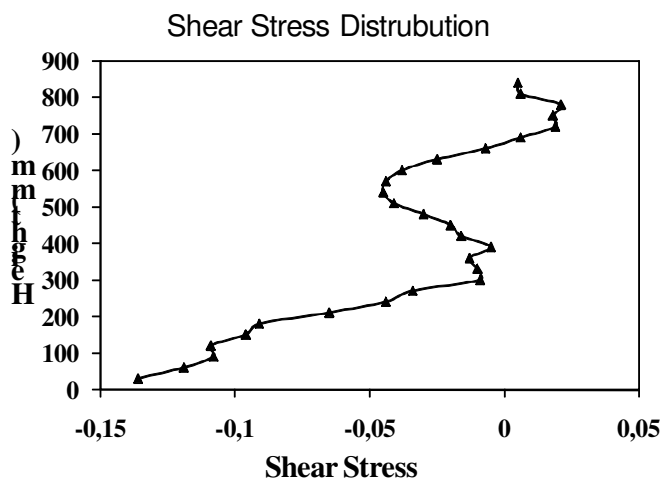

Fig. 8. Shear Stress distribution

\subsection{Open circuit wind tunnel}

During 2005 begun the design and building of a bigger turbulent boundary layer wind tunnel, open circuit one. The aim was to have a wind tunnel capable to improve, regarding the previous closed circuit one, the experimental simulation of wind conditions at the surface layer. Also, the aim to build this new tunnel as open circuit model was to have the possibility to simulate dispersion plumes and any other flow condition related with atmospheric pollution.

Such wind tunnel is $24 \mathrm{~m}$ length, which include the entrance nozzle ( $2 \mathrm{~m}$ length), the long test section $(17 \mathrm{~m})$ with constant cross area of $2.6 \times 1.8 \mathrm{~m}^{2}$ and the $5 \mathrm{~m}$ length diffuser at which end are 9 alternating current motors (15 HP each), totalizing 135HP electric power. These motors, together, have a precise velocity control, varying frequency type. The motors suction the air from the nozzle, to obtain a uniform flow, after passing the huge honeycomb. Then, the flow is "perturbed" by vertical (equal horizontally spaced) obstacles (triangular shape) to "transform" it in turbulent one, as close as possible to the wind characteristics at the surface layer. After those obstacles, the turbulent flow passes through multiple roughness elements at the floor, before to reach the test area. In this area there is a rotating disc in order to simulate, on the models, different wind directions. The rotating disc has an electric control capable to promote, manually, a very slow rotation motion to the disc. The maximum velocity at the test section is $30 \mathrm{~m} / \mathrm{s}$. For certain details see Figures 9 to 12:

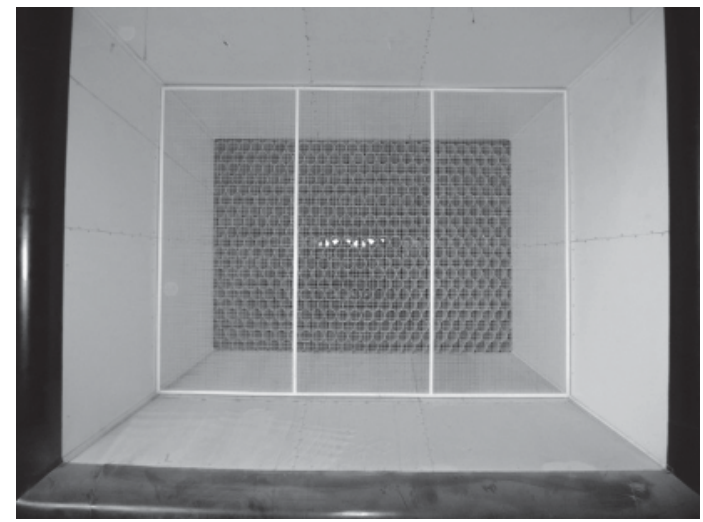

Fig. 9. Wind tunnel nozzle front view 


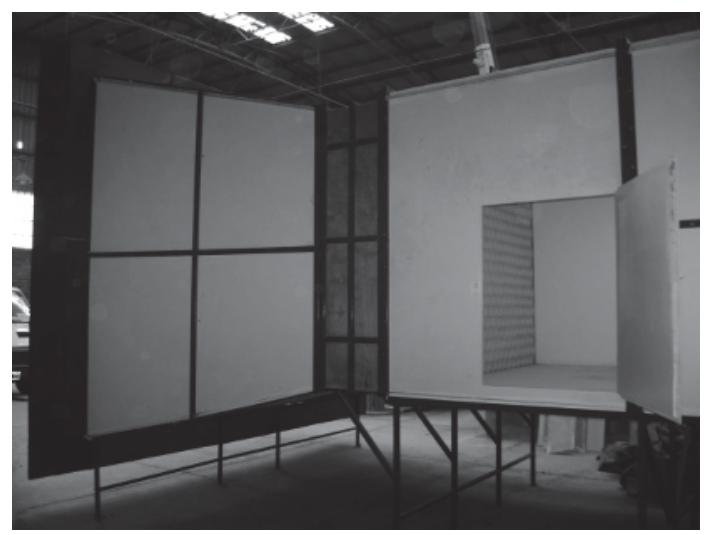

Fig. 10. Nozzle and honeycomb lateral view

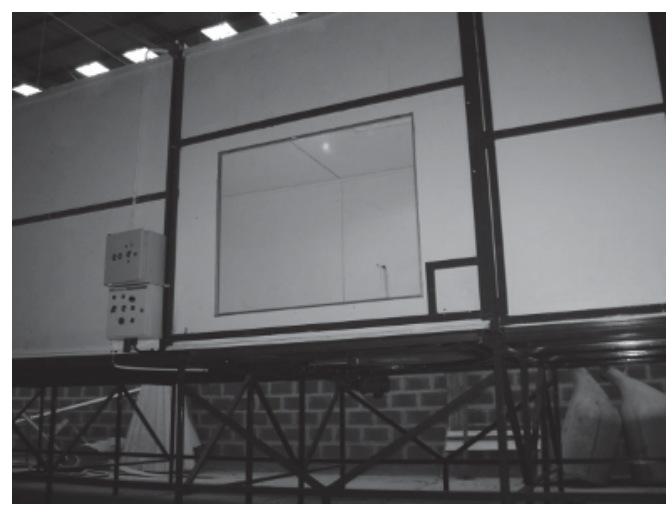

Fig. 11. Lateral external view of test section

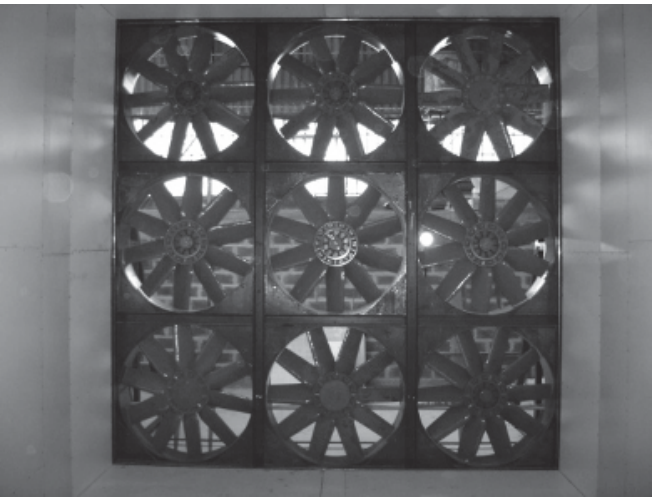

Fig. 12. Motors view from inside the diffuser 
In order to give clarity to the photos, the vertical development turbulence generators (usually located after honeycomb) and the roughness elements were removed.

At the time of the elaboration of this Chapter, authors haven't begun with experiments about turbulent flow characterization in this open circuit wind tunnel. Such step is necessary prior to plan any experimental work regarding, for example, flow control over wings. We planned to begin with the flow characterization in this tunnel on May 2011.

\section{Brief considerations about flow control}

Generally speaking we could say that flow control implies a beneficial change in the flow behavior over a body, by different passive or active devices, in comparison with such flow behavior without such devices.

The proposed tasks are various: promote the delay or early boundary layer transition; reduce or enhance the turbulence; induce or prevent separation; enhance the lift; reduce the drag; reduce the flow-induced noise.

By passive flow control we means the use of devices, for example over wings, without any movement, that's, the device acts on the flow by a passive way as an "obstacle" immersed in the flow.

By other hand, there are devices capable to move by some mechanism, acting upon the flow. Such devices could have some "feedback" being a more sophisticated active mechanism.

The authors, with the collaboration of other researchers at the Laboratory, were performed various experimental works evolving, either passive and/or active flow control devices. In the next sections we'll describe some of them.

In the following works, the experiments were carried out at the Boundary Layer and Environmental Fluid Dynamics Laboratory (LaCLyFA) closed circuit wind tunnel, at the Faculty of Engineering, National University of La Plata, Argentina. In each work there will be indicated the corresponding Reynolds number but, in all cases, corresponds to low Reynolds number aerodynamics.

\subsection{The wake asymmetry of an airfoil with a Gurney flap, and their connection with the observed lift increase. Boldes, U.; Delnero, J. S.; Marañon Di Leo, J.; Colman, J. and Camocardi, M.E.}

Abstract - The present research analyzes the asymmetry in the rolling up shear layers downstream the blunt trailing edge of airfoils with Gurney flaps as a lift enhancing mechanism. Experimental investigations relating the asymmetry of the vortex flow in the near wake region, able to distort the flow increasing the downwash of an airfoil, have been performed. We examine the lift behaviour and near wake region characteristics of the low Reynolds number airfoil HQ17 without and with Gurney mini-flaps of different lengths. The flow immediately downstream the trailing edge down to 2 miniflap lengths is explored in order to identify signs of asymmetry of the initial counter rotating vortex structures. Experimental evidence is presented showing that for typical lifting conditions the shear layer rollup process within the near wake is different for the upper and lower vortices: the shear layer separating from the pressure side of the airfoil begins its rollup immediately behind the trailing edge creating a stronger vortex while the shear layer from the suction side begins its rollup more downstream creating a weaker vortex. Aspects of a mechanism connecting the different evolution and pattern of these initial vortex structures with the lift increase due to these flaps are presented.

Experimental procedures and results discussion - In what follows, the airfoil is considered with the suction and pressure surfaces located above and below respectively. The basic tested model was an untwisted wing with a rectangular platform with a chord length of $45 \mathrm{~cm}$ and a span of $80 \mathrm{~cm}$. Each model with the different Gurney flaps was horizontally placed in the test section (1.4 
$x 1 \mathrm{~m}^{2}$ ). The wing was examined within the range of -12 degrees and +24 degrees of angle of attack. Airfoils with miniflaps with similar dimensions have been studied by numerical simulations [Schatz et al, 2004]. The lift and drag of a low Reynolds number airfoil HQ17 without and with Gurney flaps of four different lengths: $1 \%, 1.5 \%, 2 \%$ and $2.5 \%$ of the wing chord have been measured. Simultaneously the near wake vortex region was explored in order to recognize the initial location of the region in which the detached shear layers start to rollup, and the strength and features of the generated vortices.

Lift and drag data were acquired by an aerodynamic two components balance, built by the authors according to [Tusche, 1984], based on strain-gages type cells, arranged as a double Wheatstone bridge. Horizontal and vertical loads were measured simultaneously [Delnero et al, 2005].

Velocities were acquired by means of a six channel Dantec Streamline constant temperature anemometer, using an X-wire Dantec sensor probe 55R51 at an acquisition frequency of 2000 $\mathrm{Hz}$ per channel. The data was processed by a Vishay series 2310 signal conditioners and amplifiers. Due to the minimal frontal area of the wing sections $\left(0.8 \times 0.10 \mathrm{~m}^{2}\right)$, no blockage correction was applied to the results. Temperature was continuously measured in order to adjust the air density. Turbulent velocities were acquired, at the free stream (upstream of the model), in order to characterize the upcoming flow. Also, measurements were made downstream of the trailing edge along a grid with two horizontal points placed at distances of $2 \%$ and $4 \%$ of the airfoils chord and 13 vertical intervals of $2 \mathrm{~mm}$ (see, for details, Figure 14 ).

By analogy with the flow behind usual blunt bodies the width of the blunt trailing edge, which coincides with the length of the mini-flap $\mathrm{H}$, was taken as a significant scale of the motion in the near wake region. The leading edge of the miniflap is attached to the trailing edge of the airfoil.

Figure 13 shows the schema of a Gurney mini-flap configuration and the anemometer sensor location. Figure 14 shows the grid measurement schema.

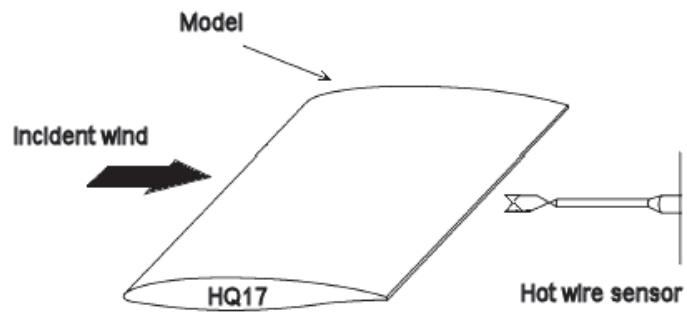

Fig. 13. Experimental setup

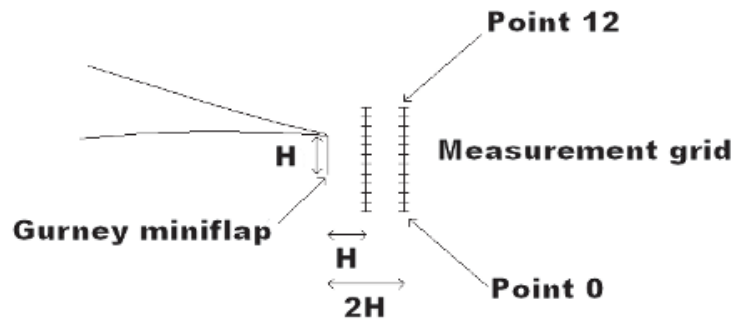

Fig. 14. Measurement grid details 
Figures 15 and 16 shows the $C_{L}$ and $C_{D}$ obtained values, plotted as a function of the angle of attack, for the plain wing and for the different miniflaps sizes. We could say that all the miniflaps increase the lift and drag coefficients, in comparison with the clean airfoil. From the drag point of view, for all the Gurney sizes, a little bit less drag is exhibit by the smaller one (1\%c).

In order to obtain more accurate information about the scale of the turbulent structures which appear intermittently in the flow downstream the trailing edge, a wavelet analysis was performed. This procedure retains information in time domain as well as in the frequency domain. The wavelet analysis of the velocity data allows the identification of aspects of turbulent structures which can be connected to transport events. The continuous wavelet transform used in this paper, is known to be appropriate for analyzing turbulent flow data [Farge, 1992], [Farge, 1990].

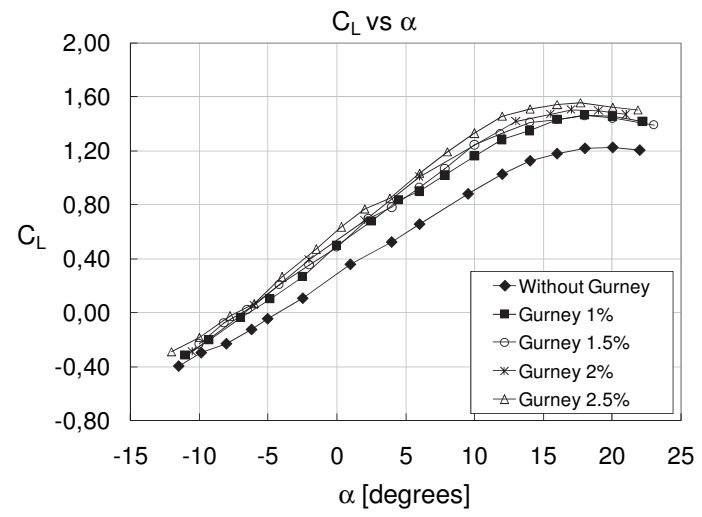

Fig. 15. $\mathrm{C}_{\mathrm{L}}$ vs angle of attack

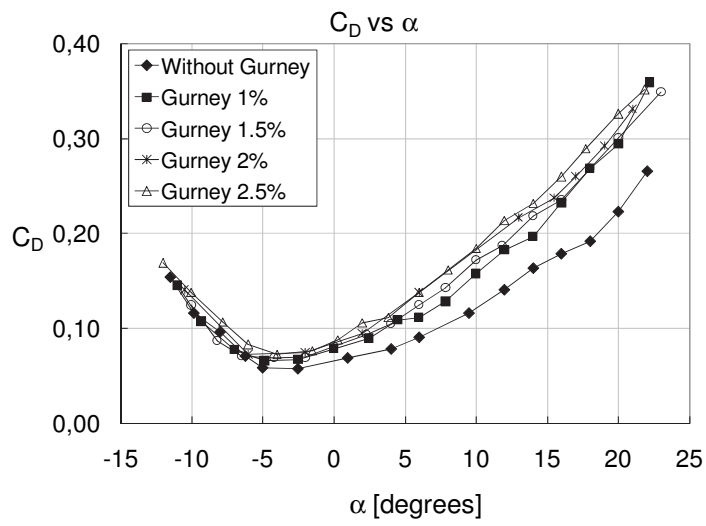

Fig. 16. CD vs angle of attack 
Our aim was to compare time scales, intensities and frequencies of the turbulent structures in the wavelet map behind the Gurney flap. Some wavelet interpretation criteria used by Mahrt (1991), estimating the time extent and frequency of a particular detected structure directly from its wavelet graph scale. The velocity-time records were explored in order to detect features related to the second derivative of a Gaussian g2 (usually called the "Mexican hat" wavelet). Assuming frozen flow theory, one can deduce the Turbulent Spatial Scale for the velocity component, looking for the maximum in the wavelet map.

The measured lift values are consistent with those described by Schatz et al (2004) in their computational experiments. It is also interesting to point out that these authors subscribe to our perception in considering the influence of features of the wake flow on the wing aerodynamics when they mentioned that particular characteristics of the wake had direct influence upon the increase of the section drag coefficient.

From the top and the trailing edge of a mini-flap two shear layers emerge which roll up into a pattern of alternating counter rotating vortices establishing absolute wake instability [Huerre et al, 1985]. The Karman vortex street in the wake of a cylinder exhibits this type of instability.

When an absolutely unstable scenario exists, arbitrary disturbances injected in the flow stay at or propagates upstream and/or downstream of its point of introduction. Therefore it is to be expected that the vortex structures generated behind the airfoil are able to influence upstream and downstream conditions.

Absolute instabilities can be found in laminar and turbulent flows. They are characterized by a clear peak in the spectrum of the fluctuations in the wake and its surroundings. The near wake regions were the rollup of the shear layers evolve into the initial vortical structures behind the tested airfoils display a significant peak in the spectra of the velocity fluctuations indicating a clearly identifiable absolute instability shown in Figures 17 and 18.

However we were not interested in finding the exact location of the regions where the vortices develop. In our experiments we explored the flow immediately behind the trailing edge in regions exhibiting a dominant spectral peak, with the aim to find evidences of an enduring asymmetry able to deviates the flow in the near wake region, enhancing the downwash. By exploring the near wake region searching for regions with the highest spectral peaks we found the following differences in the behavior of the shear layers separating from the suction and pressure surfaces of the airfoils.

For typical lifting conditions the shear layer rollup process within the near wake was always different for the upper and lower vortices: the shear layer separating from the pressure side of the airfoil began its rollup immediately behind the trailing edge of the mini-flap creating a stronger vortex, while the shear layer from the suction side initiated its rollup more downstream generating a weaker vortex. These results are summarized in Tables 1 and 2.

The larger intensity of the vortex generated by the rolling up of the shear layer separating from the pressure side of the airfoil found for all the flap sizes is illustrated by the spectra shown in Figures 17 and 18. Also, closer examination of the wavelet graphs displayed in Figs 19 and 20 shows that the highest strength of the vortex from the suction side is more diffused involving a larger region of the wake than the more energetic vortex generated by the shear layer separating from the pressure side of the airfoil, which exhibits its highest intensity concentrated in a smaller region. 

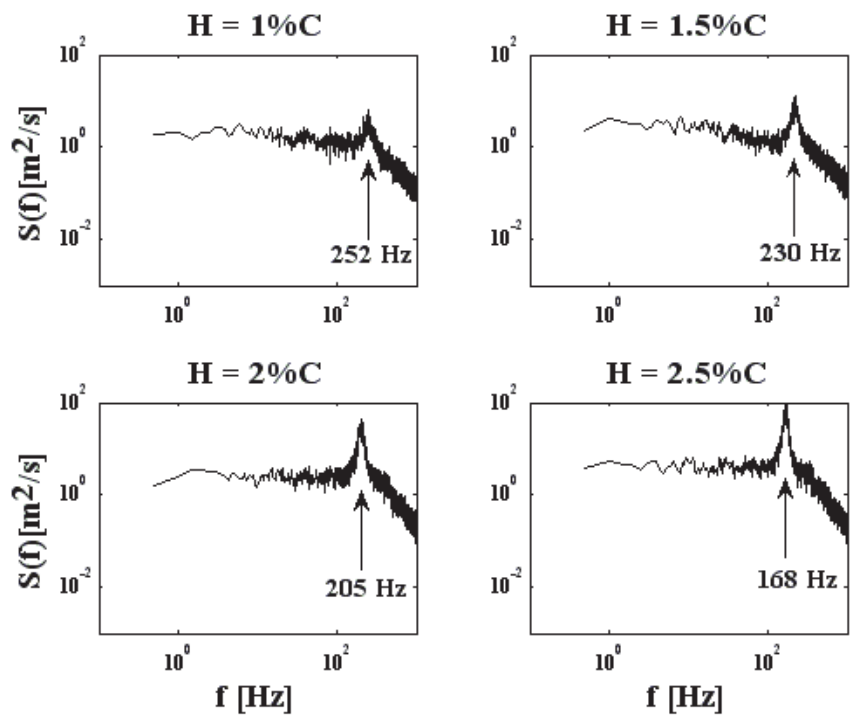

Fig. 17. Power Density Spectra Distribution for different Gurney flap sizes related to the airfoil chord $(\mathrm{C})$ at point 2 of the grid and $2 \mathrm{H}$ downstream into the wake (v component)
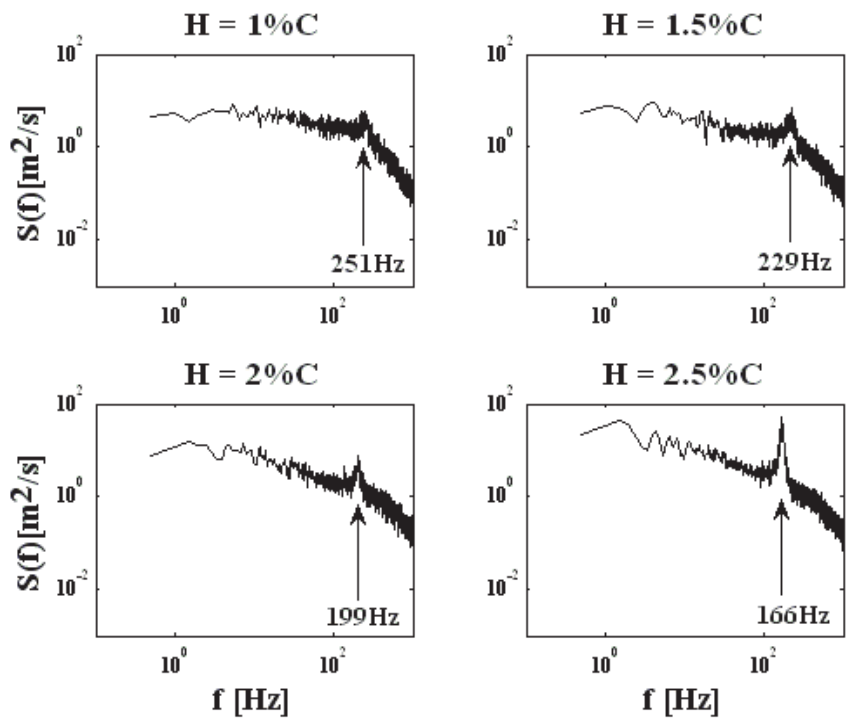

Fig. 18. Power Density Spectra Distribution for different Gurney flap sizes of the airfoil chord (C) at points 7, 8, 9, and 10 of the grid, corresponding to the trailing edge of the profile, and at $2 \mathrm{H}$ downstream into the wake ( $\mathrm{v}$ component) 


\begin{tabular}{|c|c|c|}
\hline \multicolumn{3}{|c|}{$1 \%-1 \mathrm{H}$} \\
\hline Point & Frequency $[\mathrm{Hz}]$ & $\mathrm{S}(\mathrm{f}) \mathrm{v}\left[\mathrm{m}^{2} / \mathrm{s}\right]$ \\
\hline 2 & 251 & 5,7 \\
\hline 7 & 251 & 4,46 \\
\hline \multicolumn{3}{|c|}{$1,50 \%-1 \mathrm{H}$} \\
\hline Point & Frequency $[\mathrm{Hz}]$ & $\mathrm{S}(\mathrm{f}) v\left[\mathrm{~m}^{2} / \mathrm{s}\right]$ \\
\hline 2 & 215 & 7,32 \\
\hline 8 & 229 & 6,15 \\
\hline \multicolumn{3}{|c|}{$2 \%-1 \mathrm{H}$} \\
\hline Point & Frequency $[\mathrm{Hz}]$ & $\mathrm{S}(\mathrm{f}) \mathrm{v}\left[\mathrm{m}^{2} / \mathrm{s}\right]$ \\
\hline 2 & 199 & 28,5 \\
\hline 9 & 203 & 4,14 \\
\hline \multicolumn{3}{|c|}{$2.5 \%-1 \mathrm{H}$} \\
\hline Point & Frequency $[\mathrm{Hz}]$ & $\mathrm{S}(f) v\left[\mathrm{~m}^{2} / \mathrm{s}\right]$ \\
\hline 2 & 167 & 146 \\
\hline 10 & 169 & 45,3 \\
\hline \multicolumn{3}{|c|}{}
\end{tabular}

Table 1. Power Density Spectra Peaks and peak Frequency for Gurney flaps of different sizes, at the downstream distances $\mathrm{H}$.

\begin{tabular}{|c|c|c|}
\hline \multicolumn{3}{|c|}{$1 \%-2 \mathrm{H}$} \\
\hline Point & Frequency $[\mathrm{Hz}]$ & $\mathrm{S}(\mathrm{f}) v\left[\mathrm{~m}^{2} / \mathrm{s}\right]$ \\
\hline 2 & 252 & 7,36 \\
\hline 7 & 251 & 5,96 \\
\hline \multicolumn{3}{|c|}{$1,50 \%-2 \mathrm{H}$} \\
\hline Point & Frequency $[\mathrm{Hz}]$ & $\mathrm{S}(f) v\left[\mathrm{~m}^{2} / \mathrm{s}\right]$ \\
\hline 2 & 230 & 13,6 \\
\hline 8 & 229 & 6,39 \\
\hline \multicolumn{3}{|c|}{$2 \%-2 \mathrm{H}$} \\
\hline Point & Frequency $[\mathrm{Hz}]$ & $\mathrm{S}(\mathrm{f}) v\left[\mathrm{~m}^{2} / \mathrm{s}\right]$ \\
\hline 2 & 205 & 53,4 \\
\hline 9 & 199 & 8,5 \\
\hline \multicolumn{3}{|c|}{$2.5 \%-2 \mathrm{H}$} \\
\hline Point & Frequency $[\mathrm{Hz}]$ & $\mathrm{S}(f) v\left[\mathrm{~m}^{2} / \mathrm{s}\right]$ \\
\hline 2 & 168 & 117 \\
\hline 10 & 166 & 55,7 \\
\hline
\end{tabular}

Table 2. Power Density Spectra Peaks and peak Frequency for Gurney flaps of different sizes, at the downstream distances $2 \mathrm{H}$. (Points 7, 8, 9 and 10 are located at the trailing edge level of the airfoil for each Gurney flap size and Point 2 always corresponds to the level indicating the trailing edge of the Gurney flap). It can be seen that the spectral peak $S(f)$ of the flow behind the trailing edge of the mini-flap is always larger than the corresponding peak behind the leading edge of the mini-flap 

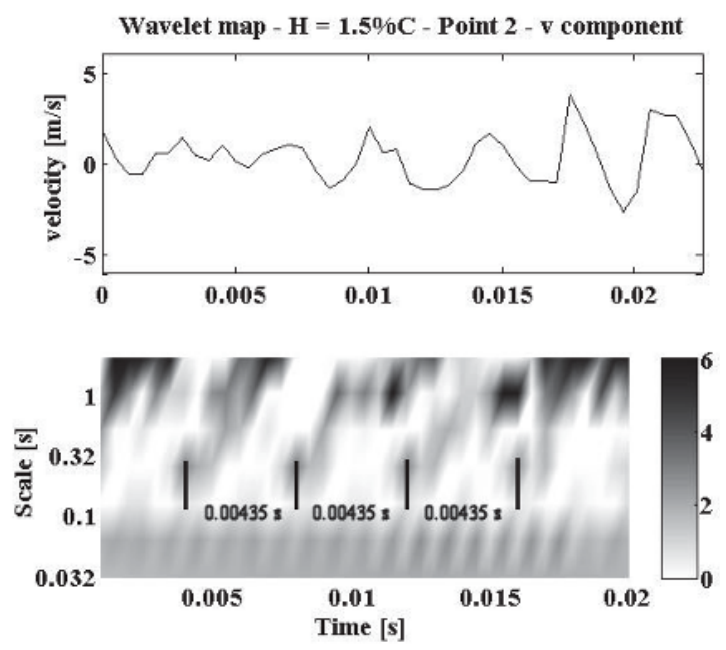

Fig. 19. Wavelet map for the Gurney flap of $1.5 \%$ of the airfoil chord $(\mathrm{H})$ at point 2 of the grid and $2 \mathrm{H}$ downstream into the wake (v component). It shows the scale of the structures, period of $0.00435 \mathrm{~s}$.

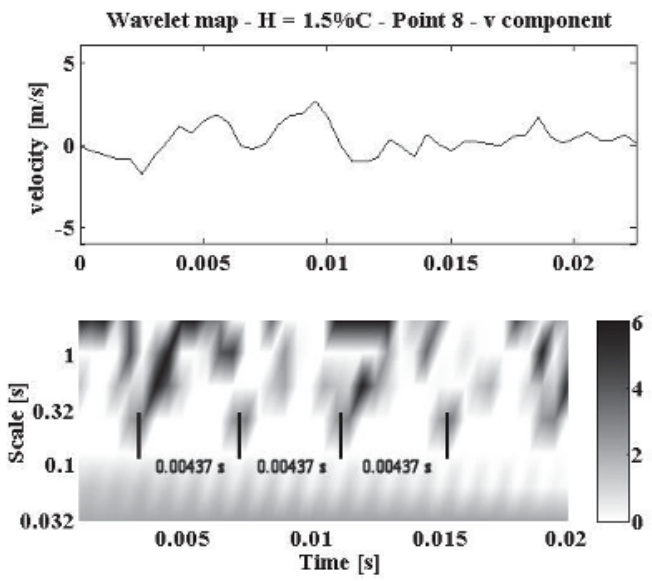

Fig. 20. Wavelet map for the Gurney flap of $1.5 \%$ of the airfoil chord $(\mathrm{H})$ at point 8 of the grid and $2 \mathrm{H}$ downstream into the wake, (v component). It shows the scale of the structures, period of $0.00437 \mathrm{~s}$.

It seems reasonable to infer that the increased strength of the lower vortex and its proximity to the downwind surface of the mini-flap deflects the location of the free rear stagnation point.

Due to the asymmetry of the initial vortices the mean location of this stagnation point is slightly shifted downstream and downward in comparison with the mean position of the stagnation point behind a blunt body with a classic Karman vortex street. 
Conclusions - How can we explain the asymmetric behavior of the initial vortices? Existing research shows that the shear layer separating from the pressure side of the airfoil is influenced by the intermittent shedding of the vortex structures originated along the upstream surface of the mini-flap. Such structures have been recently visualized [Trolin, 2006].

These authors carried out detailed time-resolved PIV visualizations of the flow around an airfoil with Gurney flap. They reported the presence of a new mode, not described in previous experimental or numerical works, in addition to the known Karman type vortex shedding mode. They showed that this mode was originated by the intermittent shedding of fluid from the upstream side of the gurney flap which interacts with the Karman type vortex wake. They suggested that this interaction could be part of a mechanism responsible for a significant portion of the overall lift increment. Such observations are qualitatively consistent with a simple interpretation of a mini-flap acting as a small barrier behaving as a passive perturbation device.

The intermittent shedding of vortex structures from the upwind region of the mini-flap into the wake reported [Trolin, 2006], can be interpreted as a perturbation mechanism acting on the shear layer emerging from the pressure side of the airfoil at its separation point from the trailing edge. The shear layer detaching from the suction side is submitted to a very different perturbation. Therefore it is to be expected that the corresponding vortex structures generated by the rollup of the shear layers will also be different introducing asymmetry in the vortex pattern of the near wake region.

It is known that turbulent shear flows are very sensitive to small changes in initial or boundary conditions and to different types of perturbations applied during transition [Oster et al, 1982]. Such perturbations could generate particular perturbation dependent shear layer organized structures that dominate the downwind evolution of the layer [Ho et al, 1985].

The mentioned shedding of the vortex structures generated in the gap upstream of the miniflap into the wake region were the shear layer is generated, fulfill the conditions of a specific perturbation acting on the region were the shear layer is generated and therefore able to influence the shape and evolution of vortex structures. The studies of Kiya et al $(1986 ; 1999 \mathrm{a}$ and 1999) describe interesting aspects of the response of a shear layer perturbed by vortices which also corroborate the asymmetric behavior detected in our experiments.

\subsection{Experimental study of a NACA 4412 airfoil with movable Gurney flap Camocardi, M.; Marañón Di Leo, J.; Delnero, J. S. \& J. Colman}

Abstract - A NACA 4412 airfoil was tested, in a boundary layer wind tunnel, with the aim to study the effect of a Gurney flap, as an active and passive flow control device submitted to a turbulent flow field. The main objective was the experimental determination of flow pattern characteristics downstream the airfoil in the near wake. The untwisted wing model used for the experiments had $80 \mathrm{~cm}$ wingspan and $50 \mathrm{~cm}$ chord, with airfoil NACA 4412. Three different movable Gurney flap mechanisms were tested, as active devices. The flap, in all cases, was located on the lower surface at a distance, from the trailing edge, of $8 \% c$ (c airfoil chord). The Reynolds number, based upon the wing chord and the mean free stream velocity was 326,000 and 489,000. The turbulence intensity was $1.8 \%$. The models were located in the wing tunnel between two panels, in order to assure a close approximation to two-dimensional flow over the model. One was a movable up-down flap geared by an electromagnetic mechanism; from the analysis of the obtained results one could appreciate an increment of $C_{l}$ when the excitation frequency increases, in comparison with the clean airfoil. Also is observed that the $C_{l}$ values of the model with the Gurney flap fixed are something greater than the 
corresponding values for the movable Gurney condition. Regarding the $C_{d}$ behavior, it diminishes when the frequency increases, but its minimum value is something greater than the case for the clean airfoil. The others were rotating flaps, geared by an electro-mechanical system, one rotate to a $90^{\circ}$ (vertical) position, and the other rotate to a $30^{\circ}$ position. In these cases the wake pattern and pressure values near the trailing edge were measured. The results obtained, for these mechanism, show us that the oscillating flap change the wake flow pattern, alleviating the near wake turbulence and enhancing the vortex pair near the trailing edge at the flap level and below that level, magnifying the effect described first by Liebeck (1978). That effect is more evident as the oscillating frequency grows. Additionally, the wake alleviation probably affects also the far wake. All of these facts suggest us to continue with the experiments, trying to measure the pressure distribution around the airfoil in all the cases, obtaining the lift and drag characteristics.

Experimental procedures and results discussion - The models were located in the wing tunnel between two panels, in order to assure a close approximation to two-dimensional flow over the model.

Figure 21 shows a diagram of how were positioned each wing model inside the wind tunnel test section.

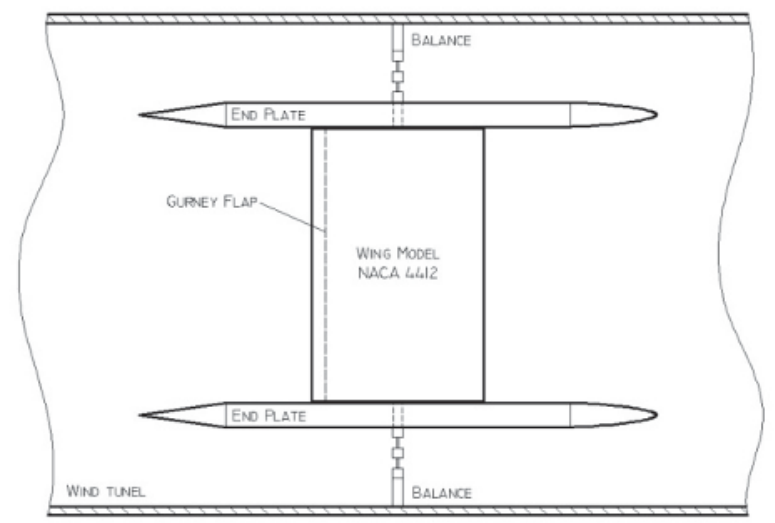

Fig. 21. Diagram of wing model inside the wind tunnel test section

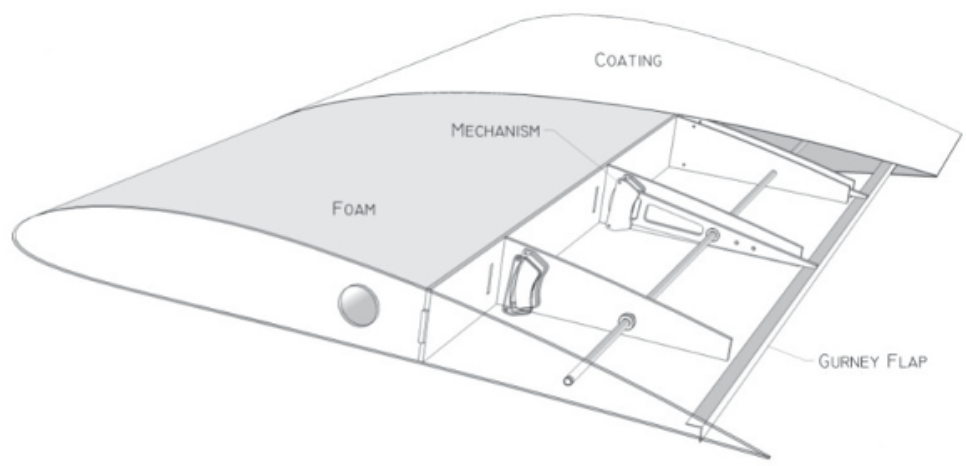

Fig. 22. Tested wing model, with vertical oscillating Gurney flap. 
The untwisted three wing model dimensions tested were $50 \mathrm{~cm}$ chord (c) and $80 \mathrm{~cm}$ wingspan, with a NACA 4412 airfoil. The Reynolds number, based upon the wing chord and the mean free stream velocity were 326.000 and 489000 , based upon the mean free stream velocity (at $1.5 \mathrm{~m}$ ahead the model at its height) and the model chord, corresponding to values of it of $10 \mathrm{~m} / \mathrm{s}$ and $15 \mathrm{~m} / \mathrm{s}$ respectively, depending on the test. The turbulence intensity was $1.8 \%$ (minimum turbulence intensity of the wind tunnel). The closest possible distance from the trailing edge, for the Gurney flap position, was determined by the implemented mechanism inside the model [Wassen et al, 2007].

The first model with the aim to study the section lift and drag coefficients behavior, when a Gurney flap located on the lower surface near the trailing edge, used as passive and active flow control device. As an active device an up-down Gurney flap was designed, geared by an electromagnetic system. Such mechanism is an electro-magnetic one excited by an electrical frequency and variable amplitude signal. By this way the Gurney flap, fixed to the seesaws (see Figure 22), was capable to make an oscillatory movement from the lower surface (0mm displacement) to the maximum amplitude $(5 \mathrm{~mm})$. The Gurney flap was a movable plate of $5 \mathrm{~mm}$ height $(1 \% \mathrm{c})$, corresponding to its maximum vertical displacement, and $1 \mathrm{~mm}$ width with a wingspan length, located on the model lower surface, at $8 \% \mathrm{c}$ from the trailing edge.

The second and third models, with the Gurney flap capable to rotate along the wingspan, were tested with the objective to measure the wake at different positions behind the trailing edge, for different rotation frequencies of the Gurney. These rotating flaps, were geared by an electro-mechanical system (see Figure 23), one rotate to a $90^{\circ}$ (vertical) position, and the other rotate to $30^{\circ}$ position. In these cases the wake pattern and pressure values near the trailing edge were measured.

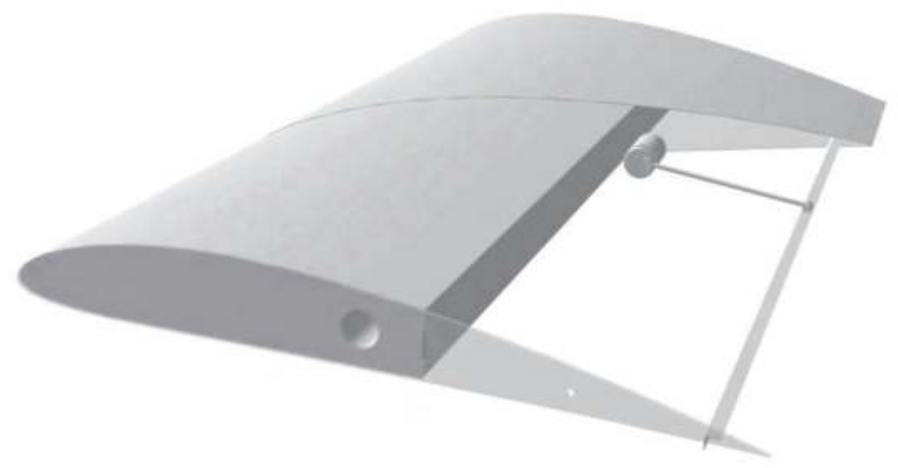

Fig. 23. Tested wing model, with rotational oscillation Gurney flap.

These models had a $10 \mathrm{~mm}$ movable plate $(2 \% \mathrm{c})$, capable to rotate along its longitudinal axe. The rotation movement, with a variable frequency, was produced by an 8 pole brushless electric motor of $28 \mathrm{~mm}$ diameter, located inside the model, actuated by a tiny frequency control, with a crankcase which transmits the rotation movement to a small brace located on the Gurney plate, closed to the longitudinal hinge of it. So, the rotation movement of the electric motor was converted in an oscillatory movement of the small Gurney plate.

The aerodynamic loads were measured by a system of two components aerodynamic balance with strain gages, with the wing model mounted built in - built in, with a built in 
mechanism to change the angle of attack. The strain gages were arranged as two complete Wheatstone bridges. The signals output were processed by a signal conditioner and a data acquisition computer. With the acquired and processed data the section coefficients were calculated and then elaborated the characteristic polar curves.

Instantaneous velocities were measured by means of a constant temperature hot wire anemometer Dantec Streamline, with X-wire sensor probes. Pressure measurements were made by mean of a Pressure System (NetScanner, equipped with piezoelectric sensors). The connection between the pressure taps and the Pressure System was by tubes of $1.8 \mathrm{~mm}$ inside diameter, each of the same length. Such pressure taps were located upstream of the perturbation zone (flap position) with the aim to obtain instantaneous pressure data not perturbed, directly, by the flap position itself. The flaps frequencies were measured with a laser tachometer.

Three different experiments were carried out, one with each Gurney flap system configuration.

Case 1: This was the case of the Gurney flap with up-down movement system. In this case the characteristic polars were obtained for a Reynolds number of 326.000, based upon the model chord and the free stream mean velocity. The experiments were divided in two steps. The first evolve tests with the clean model and with the model with the Gurney flap as passive flow control device. The second with the Gurney flap with movable capabilities, as described above. In this second step we planned to investigate the influence of the excitation frequency upon characteristic polars, for three frequency values: 5, 10 and $15 \mathrm{~Hz}$. It's necessary to point out that many technical difficulties were founded along the test, due probably to the fact that those were our first experiences with movable Gurney flaps.

Case 2: This was the case of the rotating Gurney flap (to $90^{\circ}$ position). The experiments were carried out as follows. Once mounted the wing model inside the test section, at zero angle of attack, the velocities field were measured at different positions behind the trailing edge, using an X-wire anemometer probe. We carried on, at a give Gurney rotating frequency, velocities determinations at various $y$-positions for each $x$-position, being " $y$ " the vertical axis and " $x$ " the horizontal axis. We repeated such measurements for other frequencies. The $x$-positions were two: at $2 \% \mathrm{c}$ and $75 \% \mathrm{c}$ from the trailing edge (see Figure 24). In each position, the vertical points were 30 separated each $0.4 \%$ c. The acquisition frequency was $600 \mathrm{~Hz}$, filtered at $300 \mathrm{~Hz}$. Were taken 8192 samples in each position. The Gurney's rotating frequencies were three: $26 \mathrm{~Hz} ; 35 \mathrm{~Hz}$ and $41 \mathrm{~Hz}$. Each frequency was measured by a laser tachometer.

The whole experiments evolve five steps: The first was with the wing model without Gurney; then with a fixed Gurney, at 90 degrees respect the wing chord; and the third to five with the Gurney moving at each frequency (as described above).

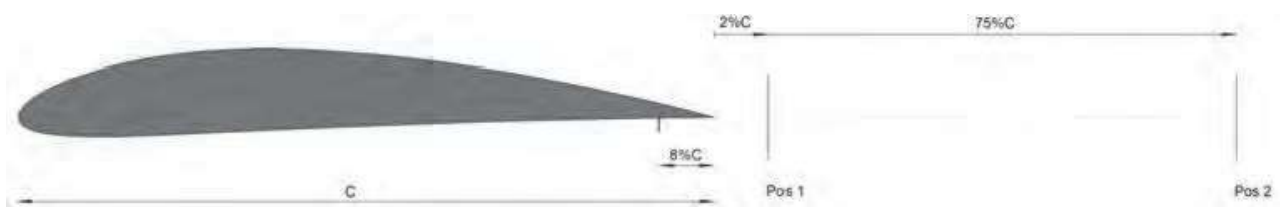

Fig. 24. Wake measurements sketch for the rotating Gurney flap at $90^{\circ}$. 
Case 3: This was the case of the oscillating Gurney flap, around its axe, up to $30^{\circ}$. The experiments were focused on the instantaneous velocity measurements in the near wake, in three " $\mathrm{x}$ " positions, $1 \mathrm{H}, 2 \mathrm{H}$ and $5 \mathrm{H}$ (Positions 1, 2 and 3 respectively, being $\mathrm{H}$ the flap height) and, at each " $x$ " position, the data were measured in 20 vertical points, which upper limit was $1.5 \%$ c and lower limit $2 \%$ c, separated each $0.04 \%$ c. Turbulence intensity was $1.8 \%$. The essays were performed for two Reynolds numbers, 326000 and 489000, based upon the mean free stream velocity (at $1.5 \mathrm{~m}$ ahead the model at its height) and the model chord, corresponding to values of it of $10 \mathrm{~m} / \mathrm{s}$ and $15 \mathrm{~m} / \mathrm{s}$ respectively.

Experiments were carried out in three steps, for each Reynolds number: $1^{\text {st }}$ step with the clean model; $2^{\text {nd }}$ step with the flap deployed but fixed (as a passive flow control device); $3^{\text {rd }}$ step with the flap oscillating at three frequencies each time $(22 \mathrm{~Hz}, 38 \mathrm{~Hz}$ and $44 \mathrm{~Hz})$. In all steps, for four values of the angle of attack: $-3^{0}, 0^{0}, 5^{0}$ and $11^{0}$ (this last near stall angle). We measured instantaneous velocities in the points cited above. The acquisition frequency was $4000 \mathrm{~Hz}$, filtered at $1000 \mathrm{~Hz}$, taken 8192 samples per channel in each measuring point. The wing model had two pressure taps at the same " $x$ " position $(0.88 \mathrm{c})$, one on the upper surface and the other on the lower surface. The connection between the pressure taps and the Pressure System was the same that in Case 2.

Figure 25 shows a schema of the wake measuring positions, along " $x$ " axis and the corresponding vertical points, indicating only the 0 and -10 points:

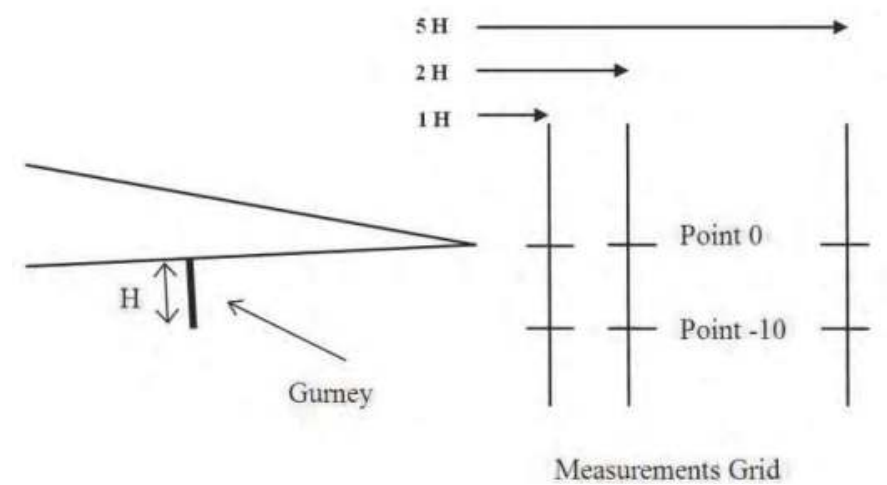

Fig. 25. Wake measurements sketch for the rotating Gurney flap up to $30^{\circ}$.

According previous works [Bacchi et al (2006), Wassen et al (2007)], we assume that a Gurney flap modify the circulation around the airfoil and, hence, its aerodynamics behavior. In this work we study such behavior on a NACA 4412 airfoil without and with a Gurney flap and, acting it as a passive and active flow control mechanism.

a. Case 1. Using the Gurney flap as a passive device we found the following. Watching the $C_{1}$ vs. angle of attack (Figure 26) is noticeable an increment of $6 \%$ of the $C_{1}$ for the model with the fixed Gurney flap in comparison with the clean wing. Also the stall angle diminishes from $14^{\circ}$ to $12^{\circ}$ and the zero lift angle of attack increase, in absolute terms, from $-3.5^{\circ}$ to $-5^{\circ}$. Comparing the $C_{d}$ vs. angle of attack curves (Figure 27), a section drag increment is observed along the curve being more important in the range of high angles of attack. Also there was a $30 \%$ increment of the $C_{d o}$ in comparison with the clean airfoil. The $C_{1}$ vs. angle of attack slope, don't show significant changes in comparison with the clean airfoil. All of these conclusions have a good agreement with other authors for different airfoils. 


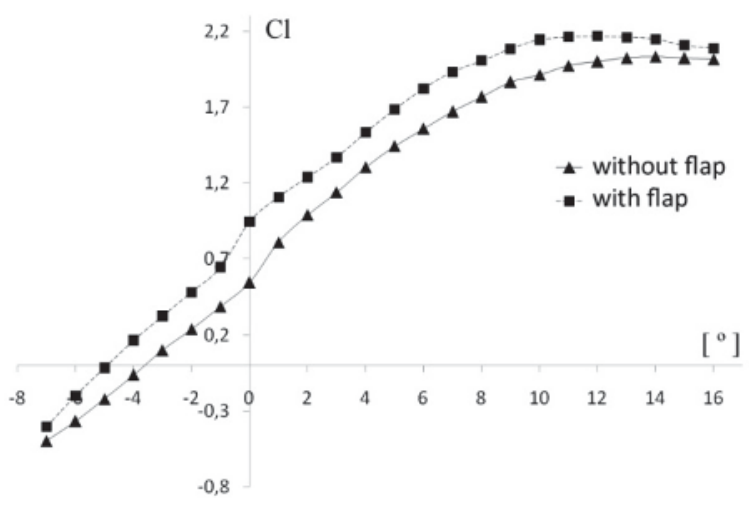

Fig. 26. Polar curve $C_{l}$ vs angle of attack

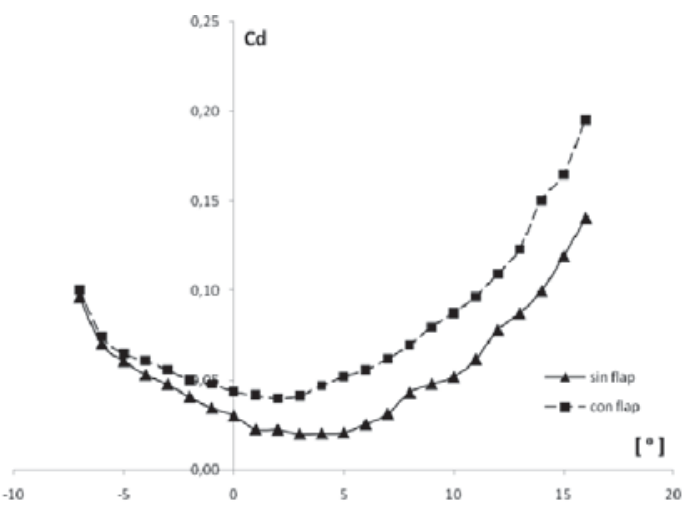

Fig. 27. Polar curve of $C_{d}$ vs. angle of attack

For the Gurney flap with vertical movement (oscillation), working as an active device, we found the following. The aerodynamic loads were measured, in a first instance, for two angles of attack, $0^{\circ}$ and $8{ }^{\circ}$, with the movable flap at 5,10 and $15 \mathrm{~Hz}$ frequencies, with the motivation to obtain preliminary results about the device behavior. Table 1 shows the section coefficients for those two angles of attack and the different excitation frequencies.

\begin{tabular}{|cccc|}
\hline Frecuency & $\alpha$ & $C_{l}$ & $C_{d}$ \\
\hline \multirow{2}{*}{$\mathbf{5 ~ H z}$} & $0^{\circ}$ & 0,499 & 0,084 \\
& $8^{\circ}$ & 1,423 & 0,173 \\
\cline { 2 - 4 } & $0^{\circ}$ & 0,523 & 0,049 \\
& $8^{\circ}$ & 1,435 & 0,160 \\
\cline { 2 - 4 } & $0^{\circ}$ & 0,524 & 0,033 \\
& $8^{\circ}$ & 1,479 & 0,150 \\
\hline
\end{tabular}

Table 3. Aerodynamic coefficients, for $0^{\circ}$ and $8^{\circ}$ at 5, 10 and $15 \mathrm{~Hz}$. 
In a second instance the curves $C_{1}$ vs. a were determined measuring loads for a range of angles of attack from $-6^{\circ}$ to the stall at approximate 17 , for the excitation frequencies of 5 and $10 \mathrm{~Hz}$ (see Figure 28).

From the analysis of such curves one could appreciate an increment of $\mathrm{C}_{1}$ when the excitation frequency increases, in comparison with the clean airfoil. Such behavior is consistent with the values of Table 1 and Figure 28. Also is observed that the $C_{1}$ values of the model with the Gurney flap fixed are something greater than the corresponding values for the movable Gurney condition. Regarding the $C_{d}$ behavior, from Table 3, it diminishes when the frequency increases, but its minimum value is something greater than the case for the clean wing.

Case 2. For the case of the rotating Gurney flap $\left(90^{\circ}\right)$ the experiments show us the following: Regarding the clean wing, there were found minor velocity variations at the wake, for the two x- positions (upstream mean velocity of $10 \mathrm{~m} / \mathrm{s}$ ). The wing with the fixed Gurney exhibit Power Density Spectrum peaks around $100 \mathrm{~Hz}$, for both x-positions.

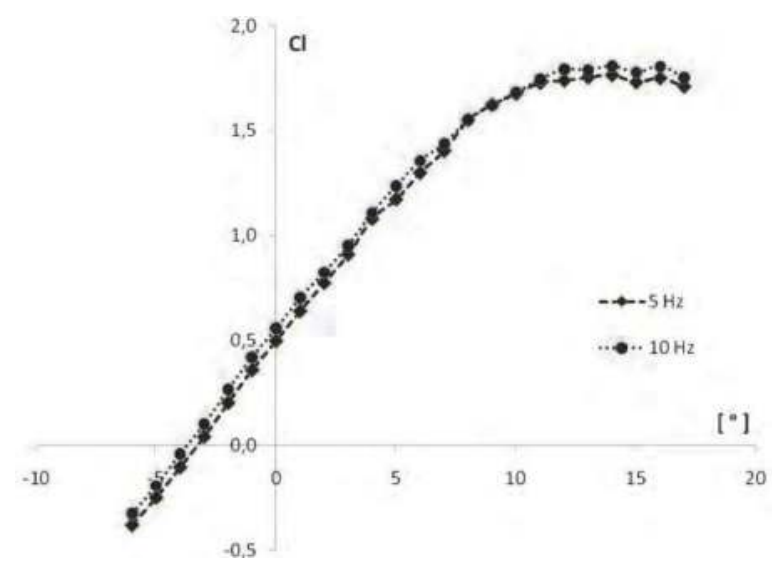

Fig. 28. $\mathrm{C}_{\mathrm{l}}$ vs. angle of attack, for excitation frequencies of 5 and $10 \mathrm{~Hz}$.

But the wing with the movable Gurney, showed such peaks, for both x-positions, at frequency values closed to the own rotating flap frequencies (see Tables 4 and 5). So, we detected a very good agreement between the rotating frequencies and the Power Density Spectrum peaks for both $\mathrm{x}$-positions at the wake.

\begin{tabular}{|l|l|}
\hline Frecuency 1 & $26 \mathrm{~Hz}$ \\
\hline Frecuency 2 & $35 \mathrm{~Hz}$ \\
\hline Frecuency 3 & $41 \mathrm{~Hz}$ \\
\hline
\end{tabular}

Table 4. Gurney flap frequency oscillations.

The example shown in Tables 4 and 5 corresponds to the second measurement position in the wake (x-position of $75 \% \mathrm{c}$ from the trailing edge), for a y-position of $2 \mathrm{~mm}$ below the trailing edge. In general, all the values are similar for both $x$-positions. Outside the wake the flow behavior is as expected. 


\begin{tabular}{|c|c|c|c|}
\hline & Frecuency 1 & Frecuency 2 & Frecuency 3 \\
\hline Position 1 & 25 & 35 & 40 \\
\hline Position 2 & 25 & 30 & 39 \\
\hline
\end{tabular}

Table 5. Power Density Spectrum peak frequencies.

In Figures 29 and 30 we show the mean v-component velocity distributions for the two measured positions (Position 1 and Position 2) and for the different Gurney flap frequency movement. MFF1P1 means: movable flap at Frequency 1 at Position 1, and then so on. Gurney $90^{\circ}$ means a fix flap at $90^{\circ}$ from the chord.

In such Figures we could appreciate the difference between the curves for the fixed Gurney and the corresponding for the moving one, for the three frequencies. At the level of the moving flap, between points 0 and -4 , the difference between velocities are very small. It seems that the vertical velocities were almost independent of the frequency in those points.

Case 3. Below we show up some of the rotating Gurney flap (up to $30^{\circ}$ ) experimental results. We displayed part of the huge instantaneous velocity and static pressure measurements, with the aim to explore qualitatively and quantitatively the particular fluid dynamic pattern, promoted by the flap oscillations, in the near wake region and their relation with the corresponding pressure distribution around the airfoil, as a consequence of the active flow control of such device upon the airfoil's aerodynamic characteristics. We observed the almost perfect matching between the flap oscillating frequencies and the special wake structure, with a peak at the same frequency than the oscillating one and other peaks which are other structures, not harmonics because they aren't multiples of the first (fundamental). In order to support such assumptions, we also showed the corresponding velocities spectra at some selected vertical points (Figure 32) and the horizontal and vertical velocities components on each " $x$ " position $(1 \mathrm{H}, 2 \mathrm{H}$ and $5 \mathrm{H})$ for all the vertical points (mean free stream of $10 \mathrm{~m} / \mathrm{s}$ and $0^{0}$ angle of attack):

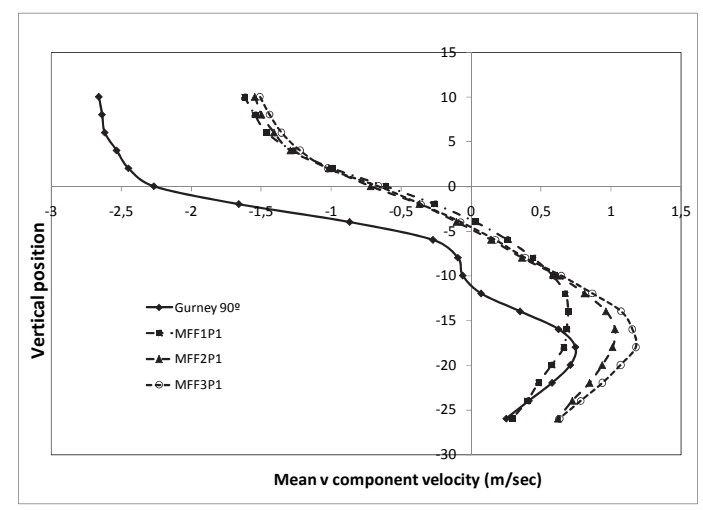

Fig. 29. Mean v-component velocity distributions along different y-positions for $2 \% \mathrm{c} x$ position 


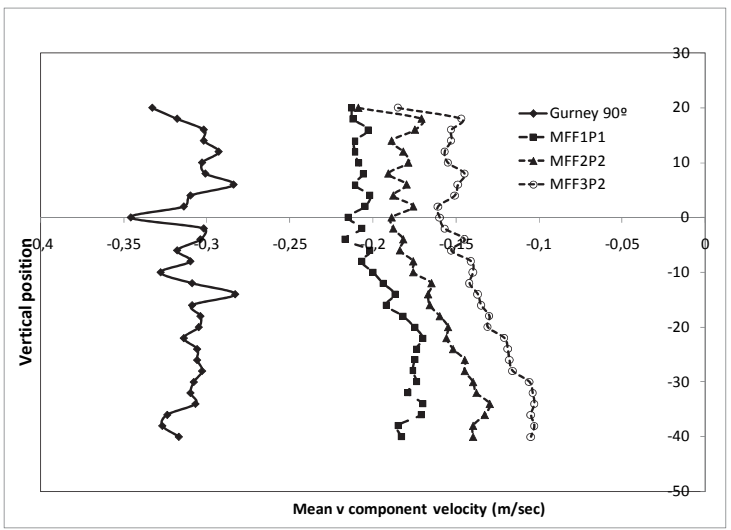

Fig. 30. Mean v-component velocity distribution along different y-positions for $75 \% \mathrm{c} x$ position

Figure 31 shows a Power Density Spectra, corresponding to the fixed Gurney, measured at $2 \%$ c $x$-position (Position 1). Figure 32 shows the power density spectra evolution of the downstream vertical velocities, at the $2 \mathrm{H}$ " $\mathrm{x}$ " position in a vertical point located at the same horizontal level of the flap's trailing edge (point -10), for the clean airfoil (PS), the flap deployed fixed (GF) and for the flap oscillating at 22; 38 and $44 \mathrm{~Hz}$. In all cases, the free stream upstream velocity was $10 \mathrm{~m} / \mathrm{s}$.

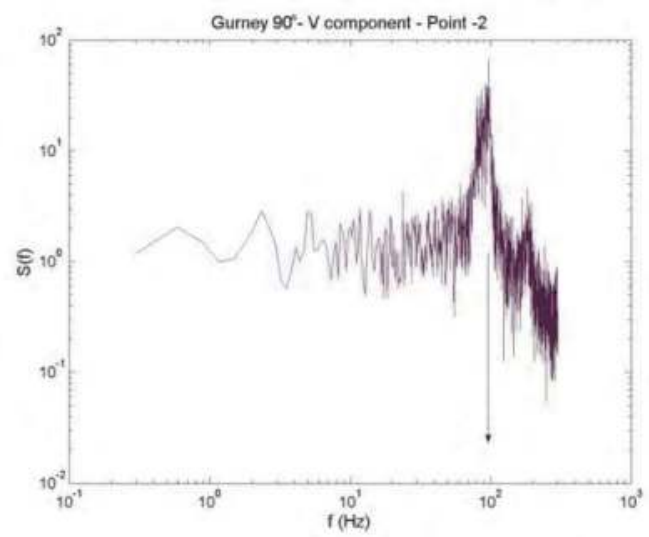

Fig. 31. Power Density Spectra for Position 1 at the trailing edge of the Gurney flap. 

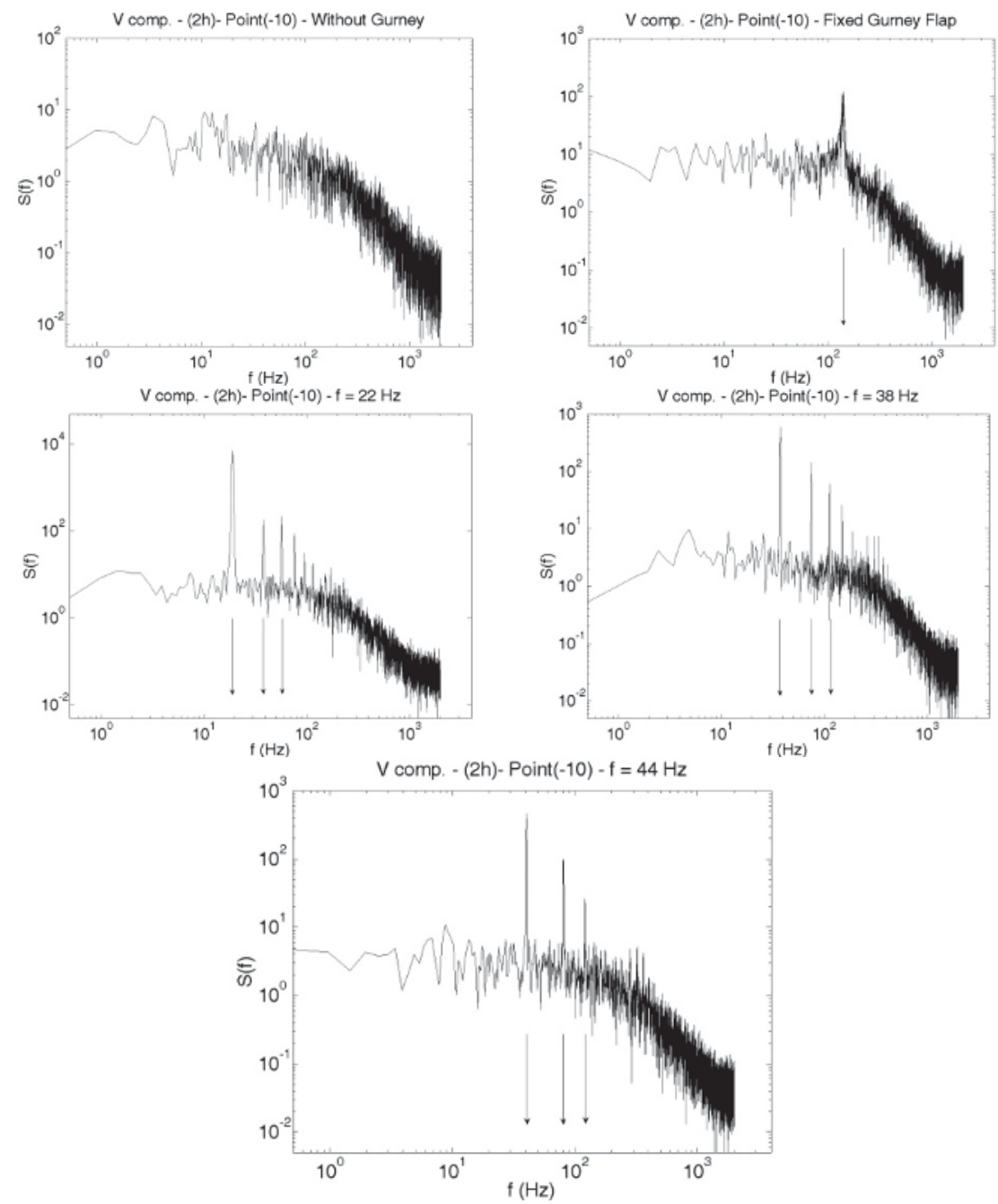

Fig. 32. V component velocities spectra, for the clean airfoil, fixed Gurney and moving one (three frequencies), 2H x-position and vertical point -10 .

The spectra peaks were as follows: For $22 \mathrm{~Hz}$ (oscillating frequency), first peak at $22 \mathrm{~Hz}$ and successively (approximate) $39 \mathrm{~Hz}, 58 \mathrm{~Hz}, 74 \mathrm{~Hz}, 92 \mathrm{~Hz}$ and $110 \mathrm{~Hz}$; for $38 \mathrm{~Hz}$ (oscillating frequency), the peaks were at $30 \mathrm{~Hz}, 78 \mathrm{~Hz}, 110 \mathrm{z}, 124 \mathrm{~Hz}, 190 \mathrm{~Hz}$; for $44 \mathrm{~Hz}$ (oscillating frequency), the peaks were $44 \mathrm{~Hz}, 82 \mathrm{~Hz}, 108 \mathrm{~Hz}$. For the fixed GF condition, the peak was at $142 \mathrm{~Hz}$. One could see how the oscillation of the flap made important changes on the wake characteristics.

One could see how the oscillation of the flap made important changes on the wake characteristics. The periodic (coherent) vortex street, generated by the oscillating flap, had enough strength to overlap and diminish the intensity of the turbulent structures typical of the airfoil with the fixed flap. This behavior is more significantly as the oscillating 
frequency grows. In that way, the important changes in the wake, promoted by the oscillating flap, will affect directly the general circulation around the airfoil. Thus, our main concern is to measure the near wake with some detail.

Figure 33 shows the instantaneous velocities at the point -10 . The curves exhibit peaks, of course, in accordance with those power density spectra (Figure 32). We could see how the turbulent intervals between peaks, are reduced as the oscillating frequency grows. This is due, probably, to the fact that once we overcome some frequency step, the characteristics of the periodic structures, shed by the oscillating flap, become almost independent of the frequency and, so, the near wake structure will be similar for frequencies above such step.

Figures 34, 35 and 36 shows the horizontal and vertical velocities, for a free stream of $10 \mathrm{~m} / \mathrm{s}$ and $0^{0}$ angle of attack, for all vertical points in each " $\mathrm{x}$ " position $(1 \mathrm{H}, 2 \mathrm{H}$ and $5 \mathrm{H})$, for the clean airfoil, the fixed deployed flap and the oscillating condition for the three frequencies.

Regarding the Figure 34, we could conclude that the U-component has small variations between the different conditions (clean airfoil; fixed flap; etc), being always positive above and below the trailing edge, but with a reduction of its magnitude from the trailing edge level to the end of flap level. The vertical velocities exhibit important differences, above the trailing edge, between the clean airfoil and the fixed flap case. Respect the oscillating flap, there are small differences between the vertical velocities for the three frequencies but, if we look close the vertical velocities at the flap level and lower, their values are greater than the corresponding to clean airfoil or even the fixed flap case. Qualitatively, the situation is similar for the $2 \mathrm{H}$ " $\mathrm{x}$ " position (Figure 35). Analyzing both Figures, it's clear that we have an anticlockwise vortex behind the flap. This is consistent with the results founded by other authors [Liebeck (1978), Neuhart et al (1988), Storms et al (1993), Bloy et al (1995), Myose et al (1998), van Dam et al (1999), Gay et al (2003), Boldes et al (2008) and Tang et al (2007)].
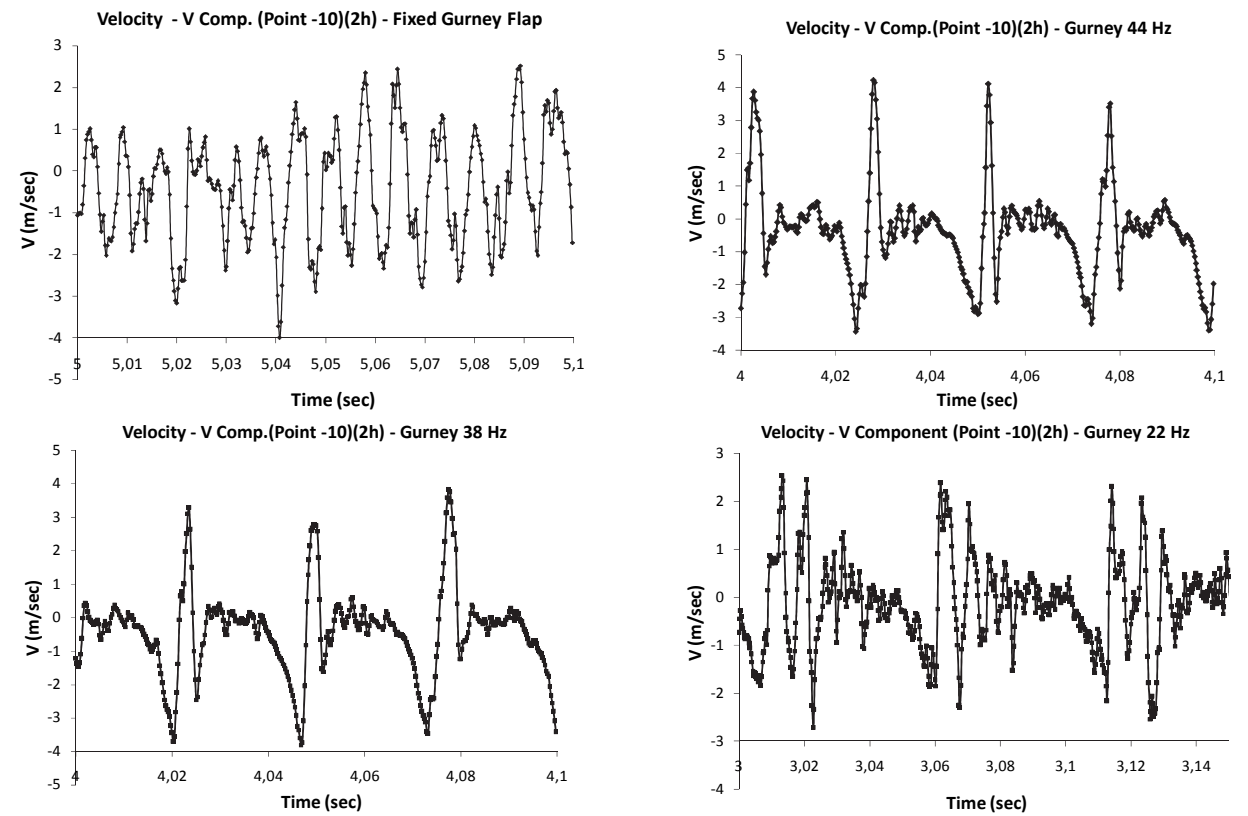

Fig. 33. Instantaneous vertical velocities curves at -10 points for the wing with GF fixed and wing for the three mini-flap frequencies oscillation 

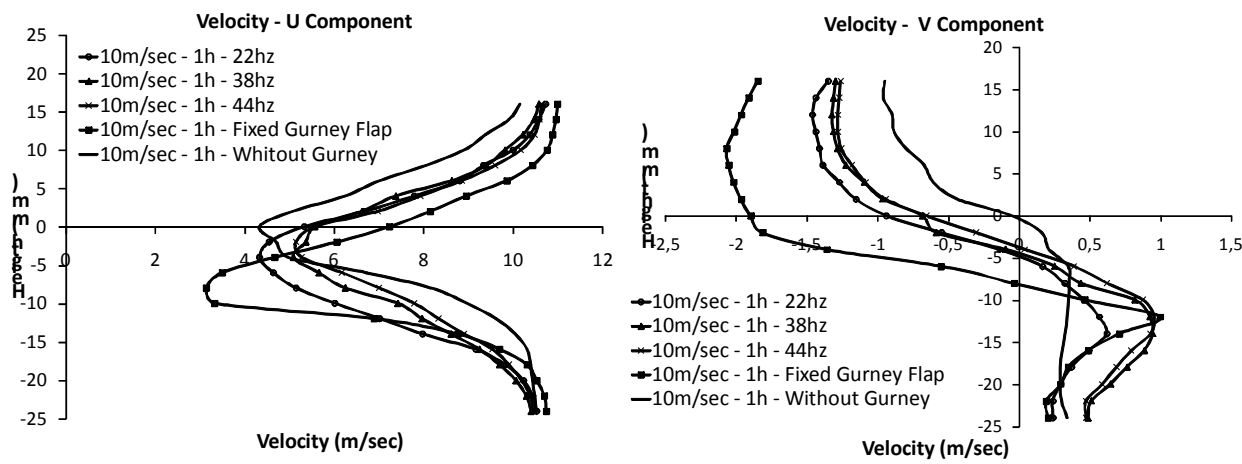

Fig. 34. U-component and V-component velocities for the fixed and movable mini-flap for different frequencies at $1 \mathrm{H} \mathrm{x}$-position
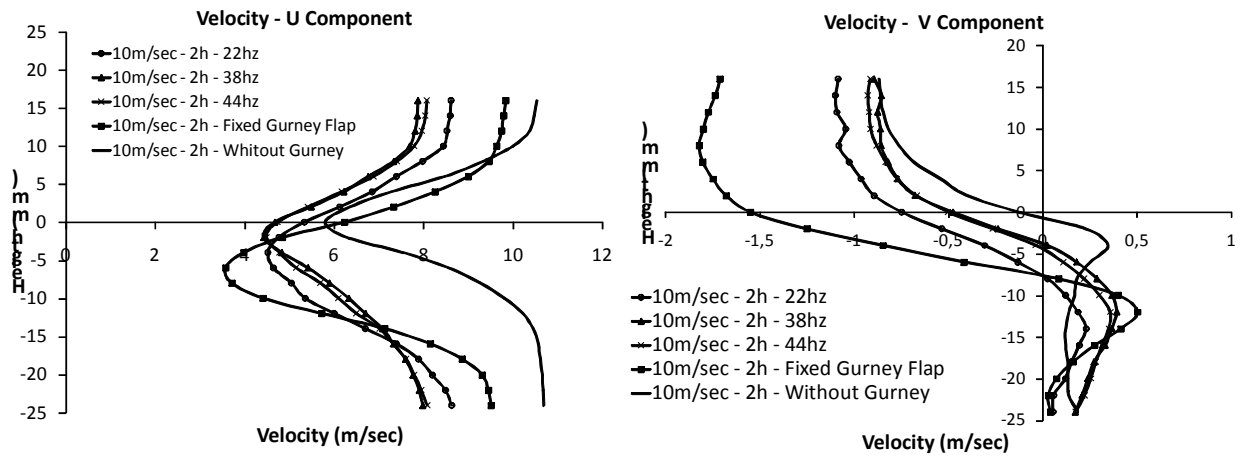

Fig. 35. U-component and V-component velocities for the fixed and movable mini-flap for different frequencies at $2 \mathrm{H}$ x-position
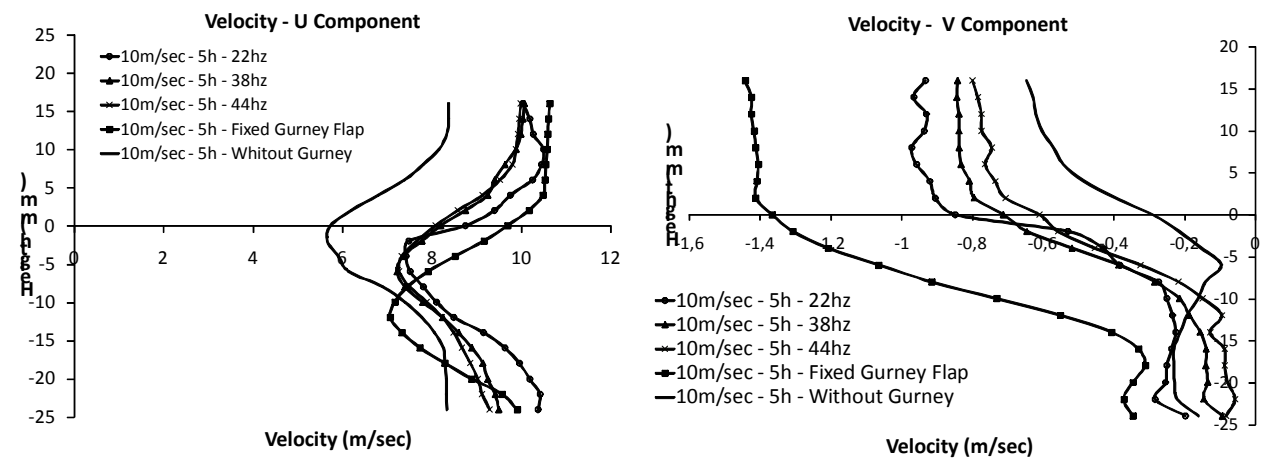

Fig. 36. U-component and V-component velocities for the fixed and movable mini-flap for different frequencies at $5 \mathrm{H} x$-position 
Following some of the ideas exposed by [Tang et al (2007)], we selected two upstream points, one on the upper surface and the other on the lower surface (see Experimental procedure), to analyze the pressure time history. Our pressure taps were located at the " $x$ " position $0.88 \mathrm{c}$ being the flap location at $0.96 \mathrm{c}$. Such pressure taps were designated, Up (for upper surface) and Low (for lower surface). The main difference, regarding the procedure followed by Tang et al [20], was our election of the pressure taps location, upstream the perturbation device (flap) location. Figures $37 \mathrm{a}$ and $37 \mathrm{~b}$ show the $\mathrm{C}_{\mathrm{p}}$ time history for $5^{0}$ angle of attack (AOA) and $22 \mathrm{~Hz}$ and $38 \mathrm{~Hz}$ oscillating frequencies, respectively. Figures $37 \mathrm{c}$ and $37 \mathrm{~d}$ are for those two frequencies but for $11^{\circ}$ of angle of attack.

Figure 37a shows some irregularities in the pressure fluctuations, than Figure 37b. It seems that as frequency grow, the pressure fluctuations become similar in amplitude, both in the upper and lower surfaces. The difference between those times histories could be associated with the changes in the near wake as the frequency grows (see Figure 33). Although velocities spectra showed in Figure 12 corresponds to $0^{0}$ of angle of attack, we could made a comparison between such results and the pressure time histories for $5^{0}$ angle of attack, bearing in mind the similar qualitative behavior of the airfoil for $0^{0}$ and $5^{0}$ angles of attack. Moreover, such times history behavior is also associated with the pair of vortex structures in the near wake (described above, regarding Figures 34 and 35).
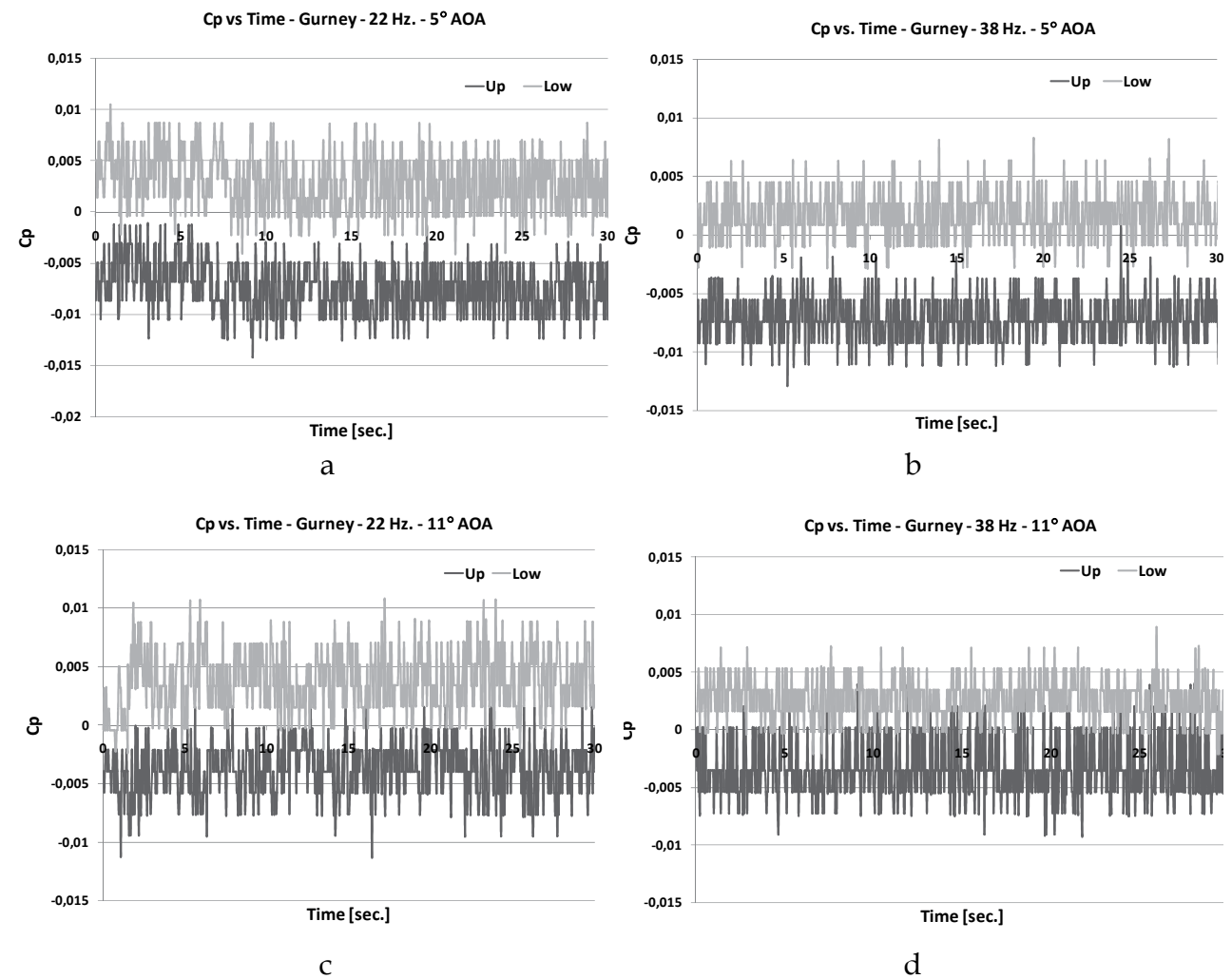

Fig. 37. Cp vs. time 
If we observe carefully, for the same angle of attack and far away from the stall, as frequency grow, the upper $C_{p}$ becomes more negative whereas the lower $C_{p}$ becomes a bit less positive as the frequency grow. From an overall point of view we could conclude that as frequency grows the lift will enhance. Figures $17 \mathrm{c}$ and $17 \mathrm{~d}$ show us the situation for $11^{0}$ of angle of attack, exhibiting an overall increase of the pressure fluctuations, in comparison with the case for $5^{0}$ of angle of attack, but with they seems to diminish the difference between the upper and lower $C_{p}$ `s. So, that could imply a small lift lowering, in comparison with the $5^{0}$ angle of attack. Such behavior, on the upper surface, could be a result of the interaction of the external turbulent flow and the boundary layer near to stall and, in the lower surface, the interaction of the external flow and the fluctuations induced by the oscillating flap.

Finally, looking to achieve an overall understanding of the whole phenomena, we prepared the Table 6 in order to compare the upper and lower $C_{p}$ `s, for the airfoil with the fixed miniflap and the airfoil with the oscillating flap, for the three frequencies.

\begin{tabular}{|c|c|c|c|c|}
\hline \multirow{2}{*}{\multicolumn{3}{|c|}{ Device }} & \multicolumn{2}{|c|}{ Position } \\
\hline & & & Cp Up & Cp Low \\
\hline \multirow{3}{*}{\multicolumn{2}{|c|}{ Deployed Gurney Flap }} & $0^{\circ}$ & $-0,1673516$ & 0,2247924 \\
\hline & & $5^{\circ}$ & $-0,413925$ & 0,2588836 \\
\hline & & $11^{\circ}$ & $-0,2374264$ & 0,2957263 \\
\hline \multirow{9}{*}{ Movable Gurney Flap } & \multirow{3}{*}{$22 \mathrm{~Hz}$. } & $0^{\circ}$ & $-0,1477534$ & 0,1360552 \\
\hline & & $5^{\circ}$ & $-0,3680741$ & 0,142911 \\
\hline & & $11^{\circ}$ & $-0,1969174$ & 0,1875045 \\
\hline & \multirow{3}{*}{$38 \mathrm{~Hz}}$. & $0^{\circ}$ & $-0,0647598$ & 0,1507643 \\
\hline & & $5^{\circ}$ & $-0,3591227$ & 0,0808359 \\
\hline & & $11^{\circ}$ & $-0,1732171$ & 0,1346238 \\
\hline & \multirow{3}{*}{$44 \mathrm{~Hz}}$. & $0^{\circ}$ & $-0,0308086$ & 0,1724985 \\
\hline & & $5^{\circ}$ & $-0,3501714$ & 0,0187608 \\
\hline & & $11^{\circ}$ & $-0,2016496$ & 0,1066335 \\
\hline
\end{tabular}

Table 6. Upper and lower $\mathrm{C}_{\mathrm{p}}$ 's for the airfoil with the fixed mini-flap and with the oscillating one

Conclusions: Three NACA 4412 airfoil model were studied, in a boundary layer wind tunnel, to investigate the aerodynamic effect upon them by a Gurney flap, as passive and active flow control device. Owing this flap was located at a distance of $8 \% \mathrm{c}$, from the trailing edge, our work is reasonable compared with other works performed with the Gurney located exactly at the trailing edge [Wassen et al, 2007].

The fixed Gurney flap increase the maximum section lift coefficient, in comparison with the clean airfoil, but increasing something the section drag coefficient. These results had good agreement with Liebeck's work [1978], who concluded that increasing the flap height until $2 \% \mathrm{c}$ the drag increases. The motivation to employ Gurney flap as an active flow control device, is to found the frequency that produce the more convenient vortex shedding from the point of view of reinforcing the airfoil circulation. If the device is fixed, in some instances the vortex shedding is favorable to enhance the lift but in other instances is unfavorable. But moving the flap, we could find the more adequate frequency in the sense to be favorable to increase the circulation and, hence, the airfoil's lift. 
In the first model for excitation frequencies up to $15 \mathrm{~Hz}$, the section lift coefficient grows meanwhile the section drag decreases. According other works [Liebeck (1978), Neuhart et al (1988)], the vortex wake close to the trailing edge, had clockwise and counterclockwise vortices. If the movable (vertical) Gurney flap oscillates outside and inside the wing, with a frequency that allows moving down the rear stagnation point of the airfoil, the lift will grow. So, according the flap frequency, it will promote an increase or decrease of the lift. Such changes are reflected in the $C_{l}$ and $C_{d}$ table shown. The main disadvantage of these experiments is to build a reliable mechanism capable to produce frequencies similar to that corresponding to the shedding vortex frequencies from a fixed Gurney flap, and also the calibration of such mechanism.

We worked at the same time in a different approach to get a movable Gurney flap, capable to reach higher frequencies, using a rotating plate of the same height of the Gurney flap used in the other system. Finally we reach a reliable mechanism, as was described above, which works at higher frequencies than the other one.

Regarding the rotating system (mini-flap to $90^{\circ}$ ), we observed a very good agreement between the Gurney rotation frequencies and the peak frequencies detected in the wake, for both x-positions (Position- 1 and Position-2, at 2\%c and 75\%c behind the trailing edge). The instantaneous velocities at the wake were measured by hot-wire constant temperature anemometer. Another noticeable fact is the difference in the vertical velocities components, between the fixed and the movable (rotating) Gurney, at both x-positions at the trailing edge height. Such vertical velocities are of less magnitude for the movable Gurney case than for he fixed one. Vertical velocities are directly connected with the drag and, so, we could presume that the drag of the wing, with the rotating Gurney, will be less than the corresponding to the fixed Gurney.

In the third case, rotating Gurney flap, up to $30^{\circ}$, the periodic vortex street had enough strength to overlap and diminish the intensity of the turbulent structures typical of the airfoil with the fixed flap. This behavior is more significantly as the oscillating frequency grows. The important changes in the wake, produced by the rotating flap, will affect the general circulation around the airfoil. The differences between the vertical and longitudinal velocities, for the three frequencies, showed to us the existence of the anticlockwise vortex behind the flap.

In the case of the pressure, the $C_{p}$ differences between the lower and upper surfaces, for three reference angles of attack $\left(0^{0}, 5^{0}\right.$ and $\left.11^{0}\right)$, are greater for the fixed flap than the oscillating one. Also we observed that the corresponding $C_{p}$ differences between the lower and upper surfaces diminish as the oscillating frequency grows, but in all cases the values are lesser than the fixed flap case.

In any case, this situation will be confirmed not until we perform in future experiments, loads measurements and also pressure distribution around the airfoil. We also will perform the measurements for more $\mathrm{x}$-positions in the wake than in the present work.

Bearing in mind this is our first work with active flow control devices, in particular, the mini-flaps Gurney type, we found that a mini-flap capable to move up and down at different frequencies, seems to enhance the lift regarding the clean airfoil and the case with such mini-flap fixed. Nevertheless those are primary assessments which should be object of future and more elaborated experiments. For other side, in order to test the mini-flap with a different kind of movement, we build a model with such mini-flap capable to make an 
oscillating motion around its axe (along wingspan). Such device could oscillate with $30^{\circ}$ of amplitude but with higher frequencies than the former model. In this case we performed more measurements in the near wake region. The first obtained results showed us that this mini-flap produce a wake alleviation, that is, both in the near wake and probably in the far wake, but their effect upon lift enhancement was, in some way, opposite to the up-down movement mini-flap. This was an effect not predictable for us, at a first sight.

Finally, due the different results obtained from the models with mini-flaps of the same size but with different kind of motions, we are planning to go deep in our experiments looking to obtain, in all cases, the aerodynamic forces, the pressure distribution around the airfoil and more detailed near and far wake measurements. We hope to reach a better understanding of the process evolved and, then, to contribute to the practical implementation in wings and/or rotor blades of such type of active devices.

\section{Acknowledgements}

Authors wish to express, in particular, their recognition for the kindly and valuable assistance gave by Dra. Ana Scarabino - researcher at the Boundary Layer \& Environmental Fluid Dynamics Laboratory - related with wavelets data process and their fluid dynamics interpretation. Also wish to recognize the constant support to our work by the other Laboratory members.

\section{References}

Babiano, A., Boetta, G., Provenzale, A., and Vulpiani, A. (1994). Chaotic advection in point vortex models and 2D turbulence, Phys. Fluids, 6, 2465-2474.

Bacchi F., Marañón Di Leo J., Delnero J.S., Colman J., Martinez M., Camocardi M. \& Boldes U. (2006). Determinación experimental del efecto de miniflaps Gurney sobre un perfil HQ - 17. IX Reunión sobre Recientes Avances en Física de Fluidos y sus Aplicaciones. Mendoza, Argentina.

Bendat J. S. and Piersol, A. G. (1986). Random Data: Analysis and Measurement Procedures, 2nd ed. John Wiley \& Sons, New York.

Bloy, A. W., and Durrant, M. T. (1995). “Aerodynamic Characteristics of an Aerofoil with Small Trailing Edge Flaps. Journal of Wind Engineering, Vol. 19, No. 3, pp. 167172.

Boldes, U.; Delnero, J.; Marañón Di Leo, J.; Colman, J.; Camocardi, M. \& François, D. (2008). "Influencia en la sustentación, de los vórtices de la estela de un perfil con miniflap tipo Gurney". Actas 1er Congreso Nacional de Ingeniería Aeronáutica. La Plata.

Bonnet, J.P., Delville, J., Glauser, M.N., Antonia, R.A., Bisset, D.K., Cole, D.R., Fiedler, H.E., Carem, J.H, Hilberg, D., Jeong, J., Kevlahan, N.K.-R., Ukeiley, L.S., Vincendeau, E. (1998). Collaborative testing of eddy structure identification methods in free turbulent shear flows. Experiments in Fluids 25, 197-225.

Bruun, H. H. (1995). Hot wire anemometry, Oxford University Press, 507 p.

Castro, I. P. (1989). An Introduction to the Digital Analysis of Stationary Signals. Adam Hilger, Bristol 
Couder Y and Basdevant C. (1986). Experimental and numerical study of vortex couples in two-dimensional flows. Journal of Fluid Mechanics 173, pp. 225 - 251.

Daubechies I. (1992). Ten Lectures on Wavelets, Society for Industrial and Applied Mathematics.

Delnero, J.S. et al. (2005). Experimental determination of the influence of turbulent scale on the lift and drag coefficients of low Reynolds number airfoils, Latin American Applied Research. Vol 35 No.4 - pp. 183 - 188.

Dunyak J., Gilliam X., Peterson R., Smith D. (1998). Coherent gust detection by wavelet transform, J. of Wind Engineering and Industrial Aerodynamics 77 \& 78, pp 467478.

Farge, M. (1990). Transformee en ondelettes continue et application a la turbulence, Journ. Annu.Soc. Math., France, 17-62.

Farge, M. (1992). Wavelet Transforms and their applications to Turbulence, Annual Rev. Fluid Mechanics, 24, 395-457.

Finnigan J. (2000). Turbulence in plant canopies, Ann. Rev. of Fluid Mechanics 32: 519571

Fouillet Y. (1992). Contribution a le etude par experimentation numerique des ecoulements cisailles libres. Effets de compressibilite. These. Universite de Grenoble.

Gai, S. L., and Palfrey, R. (2003). “Influence of Trailing-Edge Flow Control on Airfoil Performance," Journal of Aircraft, Vol. 40, No. 2, pp. 332-337.

Hinze, J.O. (1975). Turbulence. 2nd edition, McGraw-Hill.

Ho, C.M. et al. (1985). Perturbed free shear layers, Annual Review of Fluid Mechanics, Vol. 16, pp. 365-424.

Huerre, P. et al. (1985). Absolute and convective instabilities in free shear layers, Journal of Fluid Mechanics, Vol. 159, pp. 151-168.

Hussain A. K. M. F. (1986). Coherent structures and Turbulence Journal of Fluid Mech. 173, pp303-356.

Jenkins J. M. and Watts D. G. (1968). Spectral Analysis and its Applications, Holden Day, San Francisco.

Jeong, G., \& Hussain F. (1995). On the identification of a vortex. Journal of Fluid Mechanics 285, pp: 69-94.

Jimenez, J. \& Pinelli, A. (1999). The autonomous cycle of near wall turbulence, J. Fluid Mech. $389,335-359$.

Jimenez, J. \& Simens, M. P. (2001). Low-dimensional dynamics in a turbulent wall, J. Fluid Mech. 435, 81, 91.

Kaimal J. C and Finnigan J. J. (1994). Atmospheric Boundary Layer Flows. Oxford University Press.

Kline, S.J., Reynolds, W.C., Schraub, F.A., and Runstadler, P.W. (1967). The structure of turbulent boundary layers. Journal of Fluid Mechanics, Vol. 30, pp. 741-773.

Kiya, M. et al. (1986). Vortex pairs and rings interacting with shear-layer vortices, Journal of Fluid Mechanics, Vol. 172, pp. 1-15.

Kiya, M. et al. (1999a). Turbulent elliptic wakes, Journal of Fluids and Structures, Vol. 13, pp. 1041-1067. 
Kiya, M. et al. (1999). Separation Control by Vortex projectiles, AIAA-1999-3400 - AIAA Fluid Dynamics Conference, 30th, Norfolk, VA.

Liebeck R.H. (1978). Design of subsonic airfoils for high lift. Journal of Aircraft, Vol. 15, No. 9.

Lomas C. G. (1986). Fundamentals of Hot Wire Anemometry. Cambridge University Press.

Mahrt, L. (1991). Eddy asymmetry in the sheared heated boundary layer. J. Atmos. Sci., 48, 472-492.

McWilliams, J.C., and Weiss, J.B., (1994). Anisotropic Geophysical Vortices, CHAOS, 4, 305311.

Monin, A. S., and Obukhov, A. M. (1954). 'Basic laws of turbulent mixing in the surface layer of the atmosphere', Tr. Akad. Nauk SSSR Geofiz. Inst. 24, 163-187. English translation by John Miller, 1959.

Myose, R., Papadakis, M., and Heron, I. (1998). "Gurney Flap Experiments on Airfoils, Wings, and Reflection Plane Model," Journal of Aircraft, Vol. 35, No. 2, pp. 206211.

Neuhart H. \& Pendergraft O.C. (1988). A water tunnel study of gurney flaps. NASA-TM4071.

Oster, D. et al. (1982). The forced mixing layer between parallel streams, Journal of Fluid Mechanics, Vol. 123, pp. 91-130.

Panofsky H. A. and Dutton, J. A. (1984). Atmospheric Turbulence. Models and Methods for Engineering Applications, John Wiley \& Sons, New York, 397 pp.

Raupach M. R., Finnigan, J.J. and Brunet Y (1996): Coherent eddies and turbulence in vegetation canopies: the mixing layer analogy, Boundary-Layer Meteorology 78: 351382

Robinson, S.K. (1991): Coherent motions in the turbulent boundary layer. Annual Reviews of Fluid Mechanics, Vol. 23, pp. 601-639.

Schatz, M; Guenther, B; Thiele, F. (2004). Computational Modelling of the Unsteady Wake behind Gurney-Flaps, 2nd AIAA Flow Control Conference, AIAA-2417, Portland, Oregon, USA.

Schwartz M. and Shaw L. (1975). Signal Processing, McGraw Hill, 396 pp.

Soldati, A. \& Monti, R. (2001). Turbulence Modulation and Control. Springer-Verlag, Wien.

Storms B.L. \& Jang C.S. (1993). Lift enhancement of an airfoil using a Gurney Flap and Vortex Generators. AIAA 1993-0647.

Tang, D. \& Dowel, E.H. (2007). Aerodynamic loading for an airfoil with an oscillating Gurney flap. Journal of Aircraft, Vol. 44, Nr. 4, pp. 1245-57.

Tennekes H. and Lumley, J. L. (1972). A First Course in Turbulence, MIT Press, Cambridge, Massachussets.

Troolin, D.R. et al. (2006). Time resolved PIV analysis of flow over a NACA 0015 airfoil with Gurney flap, Experiments in Fluids Vol. 41, pp. 241-254.

Tusche, S. (Interner Bericht) (1984). Beschreibung des Konstruktiven Aufbaus und Kalibrierung von 6-Komponenten-DMS Windkanalwaagen, DLR, Goettingen.

van Dam, C. P., Yen, D. T., and Vijgen, P. M. H. W. (1999). “Gurney Flap Experiments on Airfoil and Wings," Journal of Aircraft, Vol. 36, No. 2, pp. 484-486. 
Wassen E., Guenther B. \& Thiele F. (2007). Numerical and Experimental Investigation of Mini-Flap Positions on an Airfoil. Technical University Berlin, 10119 Berlin, Germany. Delnero J.S., Marañón Di Leo J., Boldes U., Colman J., Bacchi F. \& Martinez M.A.M. Departamento de Aeronáutica, Universidad Nacional de La Plata, (1900) La Plata, Argentina. 


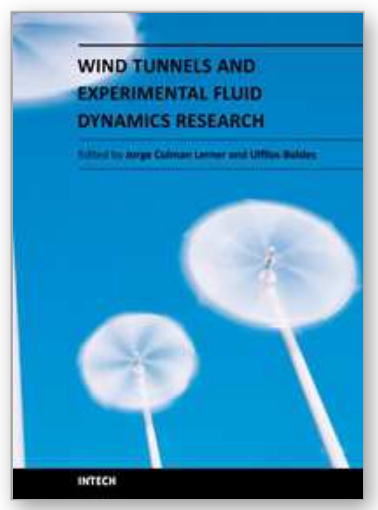

\author{
Wind Tunnels and Experimental Fluid Dynamics Research \\ Edited by Prof. Jorge Colman Lerner
}

ISBN 978-953-307-623-2

Hard cover, 709 pages

Publisher InTech

Published online 27, July, 2011

Published in print edition July, 2011

The book â€œWind Tunnels and Experimental Fluid Dynamics Researchâ€ is comprised of 33 chapters divided in five sections. The first 12 chapters discuss wind tunnel facilities and experiments in incompressible flow, while the next seven chapters deal with building dynamics, flow control and fluid mechanics. Third section of the book is dedicated to chapters discussing aerodynamic field measurements and real full scale analysis (chapters 20-22). Chapters in the last two sections deal with turbulent structure analysis (chapters 23-25) and wind tunnels in compressible flow (chapters 26-33). Contributions from a large number of international experts make this publication a highly valuable resource in wind tunnels and fluid dynamics field of research.

\title{
How to reference
}

In order to correctly reference this scholarly work, feel free to copy and paste the following:

Jorge Colman Lerner, Ulfilas Boldes, Julio Marañón Di Leo and Juan Sebastián Delnero (2011). Low Speed Turbulent Boundary Layer Wind Tunnels, Wind Tunnels and Experimental Fluid Dynamics Research, Prof. Jorge Colman Lerner (Ed.), ISBN: 978-953-307-623-2, InTech, Available from:

http://www.intechopen.com/books/wind-tunnels-and-experimental-fluid-dynamics-research/low-speedturbulent-boundary-layer-wind-tunnels

\section{INTECH}

open science | open minds

\section{InTech Europe}

University Campus STeP Ri

Slavka Krautzeka 83/A

51000 Rijeka, Croatia

Phone: +385 (51) 770447

Fax: +385 (51) 686166

www.intechopen.com

\section{InTech China}

Unit 405, Office Block, Hotel Equatorial Shanghai

No.65, Yan An Road (West), Shanghai, 200040, China

中国上海市延安西路65号上海国际贵都大饭店办公楼 405 单元

Phone: +86-21-62489820

Fax: $+86-21-62489821$ 
(C) 2011 The Author(s). Licensee IntechOpen. This chapter is distributed under the terms of the Creative Commons Attribution-NonCommercialShareAlike-3.0 License, which permits use, distribution and reproduction for non-commercial purposes, provided the original is properly cited and derivative works building on this content are distributed under the same license. 